\title{
Stability by homogenization of thermoviscoplastic problems
}

(version February 17, 2007)

\author{
Nicolas Charalambakis* François Murat
}

\begin{abstract}
.
In this paper we study the homogenization of the system of partial differential equations describing the quasistatic shearing of heterogeneous thermoviscoplastic materials. We first prove the existence and uniqueness of the solution of the system for the general model. We then define "stable by homogenization" models as the models where the equations in both the heterogeneous problems and the homogenized one are of the same form. Finally we show that three types of models, all three with non oscillating strain-rate sensitivity, are stable by homogenization: the viscoplastic model, the thermoviscous model and the thermoviscoplastic model under steady boundary shearing and body force. In these three models, the homogenized (effective) coefficients depend on the initial conditions, and, in the case of the thermoviscoplastic model, also on the boundary shearing and body force. Those theoretical results are illustrated by some numerical examples.

\footnotetext{
*Nicolas Charalambakis, Department of Civil Engineering, Aristotle University,

${ }^{\dagger}$ Laboratoire Jacques-Louis Lions, Université Pierre et Marie Curie, Boîte courrier 187,
} GR 54124 Thessaloniki, Greece ; e-mail : charalam@civil.auth.gr 75252 Paris Cedex 05, France ; e-mail : murat@ann.jussieu.fr
\end{abstract}




\section{Résumé.}

Dans cet article, nous étudions l'homogénéisation du système d'équations aux dérivées partielles qui décrit le cisaillement quasi-statique de matériaux thermoviscoplastiques hétérogènes. Nous démontrons d'abord l'existence et l'unicité de la solution du système pour le modèle général. Nous définissons ensuite les problèmes "stables par homogénéisation" comme ceux pour lequels les équations des problèmes hétérogènes et du problème homogenéisé sont de la même forme. Enfin, nous montrons que trois types de modèles (tous trois avec une sensitivité non oscillante par rapport à la vitesse de déformation) sont stables par homogénéisation : le modèle viscoplastique, le modèle thermovisqueux, et le modèle thermoviscoplastique quand les forces de volume et de surface sont indépendantes du temps. Dans ces trois modèles, les coefficients homogénéisés (effectifs) dépendent des conditions initiales et, dans le cas du modèle thermoviscoplastique, aussi des forces de volume et de surface. Ces résultats théoriques sont illustrés par des exemples numériques.

\footnotetext{
इúvoభis.

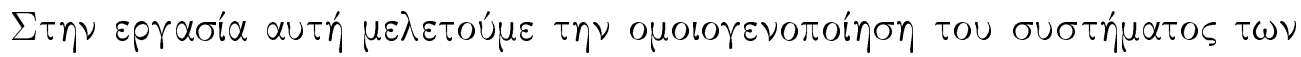

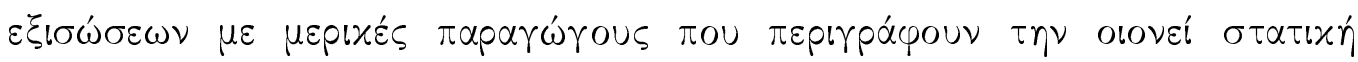

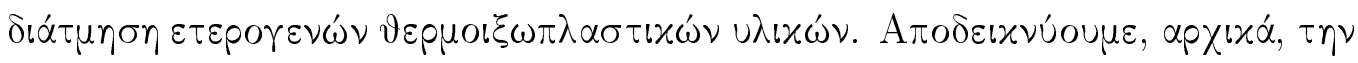

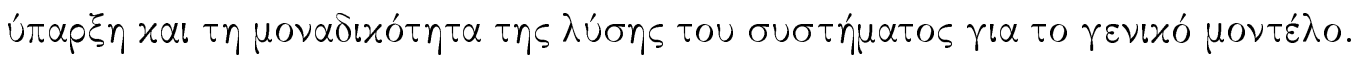

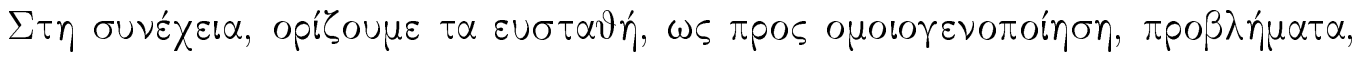

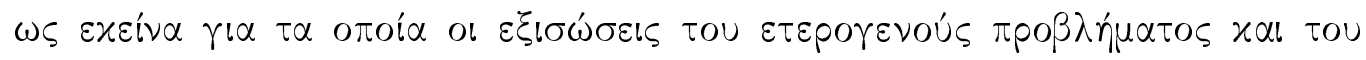

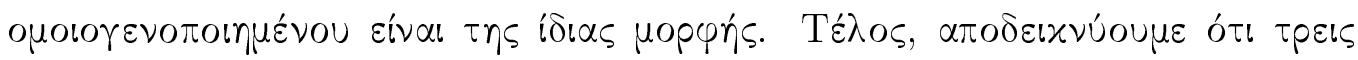

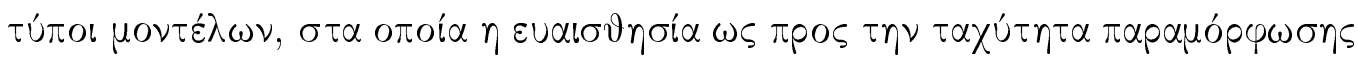

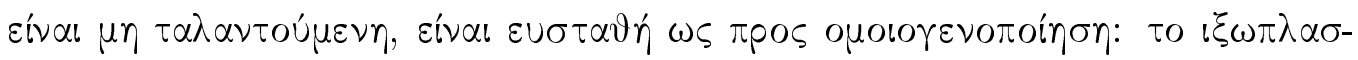

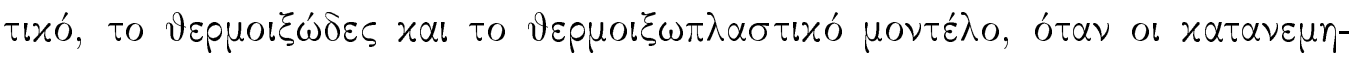




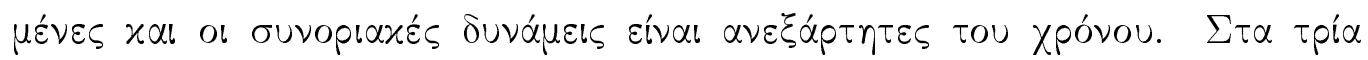

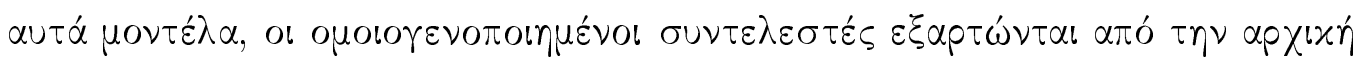

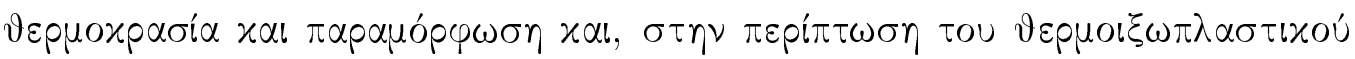

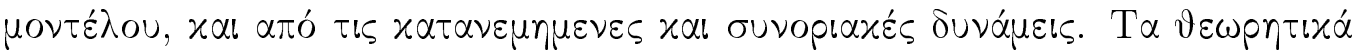






\section{Introduction}

Highly heterogeneous materials are difficult to describe, and their solutions difficult to compute numerically. Many heuristical methods have been proposed to provide "effective" equations in the hope to find a simpler model for which numerical simulations are feasible and reliable.

Mathematical homogenization provides a rigorous definition of the homogenization process and of the homogenized equation. It consists in setting the problem as a sequence of equations describing the heterogeneous material when the heterogeneities, whose typical size is characterized by a parameter $\varepsilon$, become smaller and smaller. This method, of course, assumes that the mathematical problem is well posed, or at least that one is able to prove the existence of (at least) one solution of the problem and an a priori estimate for it in some functional space. The problem is then to try to pass to the limit as $\varepsilon$ tends to zero. This task is difficult since weak topologies are involved and since passing to the limit in the problem is a nonlinear process (even if the problem is linear) when both the solution and the coefficients are concerned. This method has been proved to be successful in many cases, and we will use it in the present paper. To quote only a very few works in this direction (giving a complete list is impossible and probably more difficult than passing to the limit in any nonlinear problem) let us mention (Babuska (1976a), Babuska (1976b), Tartar (1977), Murat (1977), Bensoussan et al. (1978), Sanchez-Palencia (1978)).

In less mathematical and more mechanical words, homogenization aims at replacing a highly heterogeneous material with an equivalent (effective) one, providing a way to pass from the microscopic (oscillating) deformation to the macroscopic (slowly varying) one. This method can of course be used to "tailor" (design) new materials with enhanced properties by averaging in 
a clever (but very heterogeneous) way simple phases. It has been often described (and sometimes revisited) in the mechanical literature and here again giving a complete list is impossible; let us mention Suquet (1982), Francfort, Leguillon and Suquet (1983), Francfort, Nguyen and Suquet (1983), Hashin (1983), Suquet (1983), Maugin (1992), Aboudi et al. (1999), Ghosh et al. (2001), Bansal and Pindera (2003), Michel and Suquet (2004), Alshirts and Maugin (2005), Bansal and Pindera (2005), Bardzokas and Zobnin (2005), Guinovart-Diaz et al. (2005), Suquet (2005), Idiart et al. (2006)). In particular, the concept of "homogeneous equivalent continuum" (Maugin (1992)) has been used to predict the macroscopic response from microscopic analyses by replacing the macroscopic heterogeneous medium, represented by an element called "representative volume element", by a continuum model (see Van der Sluis et al. (1999), where a numerical homogenization technique has been proposed, which offers the possibility to determine the effective properties of a viscoplastic constitutive model).

Recent computational techniques have also been proposed, based either on a multilevel finite element method, which provides information on the evolution of the microstructure during loading (Ghosh et al. (1995), Ghosh et al. (2001)) or on an explicit coupling of the microstructural responses with the macroscopic ones (Aboudi et al. (1999)), based on volume averaging of deformation quantities and of boundary and interfacial conditions between the subvolumes used to characterize the microstructure (Aboudi et al. (1999)). Computer simulation techniques, such as multiscale dynamics plasticity (see Shehadeh et al. (2005)), merge two length scales, the nano-microscale and the continuum scale, resulting in a elastoviscoplastic model coupling discrete dislocation dynamics with finite element analyses. 
Similarly, Batra and Love (2006a) (see also Batra and Love (2006b)) analyze plane strain deformations of a representative volume element to evaluate the effective thermophysical parameters of a particulate composite made of two perfectly bonded heat conducting elastothermoviscoplastic phases. Effective values of material parameters computed in that way are compared with those obtained from existing micromechanics models and/or from the rule of mixtures. An excellent paper (Nemat-Nasser (1999)) presents a number of exact fundamental results on averaging techniques in finite deformation plasticity as well as a set of exact identities in terms of the deformation gradient and its rate and of the nominal stress and its rate.

The most obvious possibility of predicting the macroscopic response of an heterogeneous material is the choice of a macroscopic constitutive model whose material parameters are fitted onto experimental data (see Geers (1997), Meuwissen (1998), Van der Sluis et al. (1999)). The macroscopic model under consideration must then reflect some microstructural information. Although not necessary, it could be interesting to consider a model which has the same form also at the phases' level. This allows one to look for effective parameters and makes easier the mechanical characterization of heterogeneous materials. Another interesting question is whether the homogenized parameters are independent of the boundary and initial conditions.

In the present paper, we consider the test problem of simple shearing of a rigid thermovisoplastic material made of numerous layers of thickness of order $\varepsilon$ perpendicular to the $x$-direction, with different referential densities $\rho^{\varepsilon}$, specific heat coefficients $\eta^{\varepsilon}$, rates of plastic work converted into heat $\beta^{\varepsilon}$, strain-rate sensitivities $n^{\varepsilon}$ and viscosity coefficients $\psi^{\varepsilon}$. The material is supposed to be sheared uniformly by shear forces in a direction perpendicular to $x$, between two parallel planes located at $x=a$ and $x=b$. If the elastic 
effects are neglected, the balance laws of the process and the compatibility equation, which relate the unknowns of the problem (namely the velocity $v^{\varepsilon}(t, x)$, the shear stress $\sigma^{\varepsilon}(t, x)$, the strain $\gamma^{\varepsilon}(t, x)$ and the temperature $\left.\theta^{\varepsilon}(t, x)\right)$, satisfy the following system of partial differential equations, for $t \in(0, T)$ and $x \in \Omega=(a, b)$

$$
\begin{gathered}
\rho^{\varepsilon}(x) \frac{\partial v^{\varepsilon}}{\partial t}=\frac{\partial \sigma^{\varepsilon}}{\partial x}+f(t, x), \\
c^{\varepsilon}\left(x, \theta^{\varepsilon}\right) \frac{\partial \theta^{\varepsilon}}{\partial t}=\sigma^{\varepsilon} \frac{\partial v^{\varepsilon}}{\partial x}, \\
\frac{\partial \gamma^{\varepsilon}}{\partial t}=\frac{\partial v^{\varepsilon}}{\partial x}
\end{gathered}
$$

where the stress is given by the constitutive law

$$
\sigma^{\varepsilon}=\psi^{\varepsilon}\left(x, \gamma^{\varepsilon}, \theta^{\varepsilon}\right)\left|\frac{\partial v^{\varepsilon}}{\partial x}\right|^{n^{\varepsilon}(x)-1} \frac{\partial v^{\varepsilon}}{\partial x},
$$

where $\psi^{\varepsilon}\left(x, \gamma^{\varepsilon}, \theta^{\varepsilon}\right)$ is the viscosity coefficient, where $f$ denotes the body force and where the heat coefficient $c^{\varepsilon}$ is defined by

$$
c^{\varepsilon}\left(x, \theta^{\varepsilon}\right)=\frac{\rho^{\varepsilon}(x) \eta^{\varepsilon}\left(x, \theta^{\varepsilon}\right)}{\beta^{\varepsilon}\left(x, \theta^{\varepsilon}\right)} .
$$

The corresponding quasistatic problem is obtained by assuming that the inertial effects are negligible $\left(\frac{\partial v^{\varepsilon}}{\partial t} \sim 0\right)$. Then (1.1) reads as

$$
-\frac{\partial \sigma^{\varepsilon}}{\partial x}=\frac{\partial f}{\partial x}
$$

A typical form of the constitutive law (1.4) appropriate for metals is

$$
\sigma^{\varepsilon}=\mu^{\varepsilon}\left(x, \theta^{\varepsilon}\right) \nu^{\varepsilon}\left(x, \gamma^{\varepsilon}\right)\left|\frac{\partial v^{\varepsilon}}{\partial x}\right|^{n^{\varepsilon}(x)-1} \frac{\partial v^{\varepsilon}}{\partial x},
$$

where the strain-rate sensitivity satisfies $0<n^{\varepsilon} \leq 1$ and where the thermal and strain viscosity coefficients $\mu^{\varepsilon}(x, \theta)$ and $\nu^{\varepsilon}(x, \gamma)$ respectively exhibit softening and hardening behavior, i.e. satisfy

$$
\frac{\partial \mu^{\varepsilon}}{\partial \theta} \leq 0 \quad \text { and } \quad \frac{\partial \nu^{\varepsilon}}{\partial \gamma} \geq 0
$$


The above system (1.1)-(1.5) has of course to be complemented by suitable initial and boundary conditions. We note that all the (given) material functions $\rho^{\varepsilon}(x), c^{\varepsilon}(x, \theta), n^{\varepsilon}(x)$ and $\psi^{\varepsilon}(x, \gamma, \theta)\left(\right.$ or $\mu^{\varepsilon}(x, \theta)$ or $\left.\nu^{\varepsilon}(x, \theta)\right)$ are only assumed to be bounded from above and from below by strictly positive constants but are allowed to exhibit discontinuities with respect to $x$, so that equations (1.1)-(1.3) must be understood in the sense of distributions. Moreover, the heat coefficient $c^{\varepsilon}(x, \theta)$ and the viscosity coefficient $\psi^{\varepsilon}(x, \gamma, \theta)$ (or the thermal softening $\mu^{\varepsilon}(x, \theta)$ or the strain hardening coefficient $\left.\nu^{\varepsilon}(x, \gamma)\right)$ are supposed to be uniformly Lipschitz continuous with respect to $\gamma$ and $\theta$.

Homogenization consists of considering a very large number of very fine layers and of studying the limit, as $\varepsilon$ tends to zero, of the solutions of the sequence of the above problems, i.e. of describing the limit problem and the overall behavior of the velocity $v^{\varepsilon}$, stress $\sigma^{\varepsilon}$, strain $\gamma^{\varepsilon}$ and temperature $\theta^{\varepsilon}$ for fixed external forces and initial and boundary conditions. In mathematical terms, this overall behavior is expressed by the weak limits (in suitable spaces) $\left(v^{0}, \sigma^{0}, \gamma^{0}, \theta^{0}\right)$ of $\left(v^{\varepsilon}, \sigma^{\varepsilon}, \gamma^{\varepsilon}, \theta^{\varepsilon}\right)$ as $\varepsilon$ tends to zero.

If the limit problem is of the same type than the heterogeneous problems, we will say that the problem is stable by homogenization ( $\mathrm{SbH})$. More specifically, the quasistatic problem (1.2)-(1.4), (1.6) is stable by homogenization $(\mathrm{SbH})$ if the homogenized constitutive law is of the form

$$
\sigma^{0}=\psi^{0}\left(x, \gamma^{0}, \theta^{0}\right)\left|\frac{\partial v^{0}}{\partial t}\right|^{n^{0}(x)-1} \frac{\partial v^{0}}{\partial t}
$$

and if the homogenized energy equation is of the form

$$
c^{0}\left(x, \theta^{0}\right) \frac{\partial \theta^{0}}{\partial t}=\sigma^{0} \frac{\partial v^{0}}{\partial t}
$$

(compare with (1.4) and (1.2)), where $\left(v^{0}, \sigma^{0}, \gamma^{0}, \theta^{0}\right)$ are the weak limits of $\left(v^{\varepsilon}, \sigma^{\varepsilon}, \gamma^{\varepsilon}, \theta^{\varepsilon}\right)$ as $\varepsilon$ tends to zero, and where $c^{0}, \psi^{0}$ and $n^{0}$ are respectively 
the homogenized heat coefficient, the homogenized viscosity coefficient and the homogenized strain-rate sensitivity.

When $n^{\varepsilon}(x)=1$ and $\nu^{\varepsilon}(x, \gamma)=1$ in (1.7), and when (1.8) holds, i.e. in the case of the special constitutive law

$$
\sigma^{\varepsilon}=\mu^{\varepsilon}\left(x, \theta^{\varepsilon}\right) \frac{\partial v^{\varepsilon}}{\partial x}
$$

with thermal softening, the dynamical problem (1.1)-(1.4) with Dirichlet or Neumann or mixed boundary conditions has been studied by the authors (Charalambakis and Murat (1989), Charalambakis and Murat (2006a)) and its homogenization has been presented in Charalambakis and Murat (2006b). Note that in this problem the strain $\gamma^{\varepsilon}$ is defined from $v^{\varepsilon}$ by (1.3) and the corresponding initial condition, and is therefore a by-product of $v^{\varepsilon}$. In this setting, the dynamical problem (1.1)-(1.3), (1.11) is SbH, i.e. there exist an homogenized viscosity coefficient $\mu^{0}$ and an homogenized heat coefficient $c^{0}$ which satisfy (1.8) as well as lower and upper bounds and Lipschitz continuity conditions similar to the lower and upper bounds and Lipschitz continuity conditions satisfied by $\mu^{\varepsilon}$ and $c^{\varepsilon}$, such that the unique solution $\left(v^{\varepsilon}, \sigma^{\varepsilon}, \theta^{\varepsilon}\right)$ of (1.1)-(1.3), (1.11) with given boundary and initial conditions converges weakly to $\left(v^{0}, \sigma^{0}, \theta^{0}\right)$, where $\left(v^{0}, \sigma^{0}, \theta^{0}\right)$ is the unique solution of the same problem for the viscosity coefficient $\mu^{0}$, the heat coefficient $c^{0}$ and the density $\rho^{0}$. The homogenized density $\rho^{0}$ is the weak limit of the densities $\rho^{\varepsilon}$, but such is not the case for the homogenized coefficients $\mu^{0}$ and $c^{0}$, which are obtained in a much more complicated way. The homogenized (or effective) coefficients $\mu^{0}$ and $c^{0}$, which are defined explicitly through rather complicated formulas, depend only on the corresponding coefficients of the heterogeneous material and on the initial temperature. They do not depend on the other data (density, boundary conditions and initial velocity). 
Also, the homogenized coefficients $\mu^{0}$ and $c^{0}$ are independent of the type of boundary conditions (Dirichlet or Neumann or mixed boundary conditions). Finally, let us mention that in contrast with this result, the problem where the heat coefficient $c^{\varepsilon}$ only depends on $x$ is not $\mathrm{SbH}$ since in general the homogenized heat coefficient $c^{0}$ does depend on $\theta$.

In the present paper, we prove that the quasistatic problem is $\mathrm{SbH}$ if the constitutive law (1.11) (and, in some cases, the heat coefficient $c^{\varepsilon}$ ) takes special forms. More specifically, we prove that when $n^{\varepsilon}(x)=n^{\star}(x)$ and when either

(i) $\psi^{\varepsilon}(x, \gamma, \theta)=\nu^{\varepsilon}(x, \gamma)$ and $c^{\varepsilon}(x, \theta)=c^{\star}(x)$ (viscoplastic case), or when

(ii) $\psi^{\varepsilon}(x, \gamma, \theta)=\mu^{\varepsilon}(x, \gamma)$ (thermoviscous case), or finally when

(iii) $\psi^{\varepsilon}(x, \gamma, \theta)$ general but when $\sigma^{\varepsilon}(t, x)=\sigma^{\star \star}(x)$

(general thermoviscoplastic case under steady boundary shearing and body force),

the quasistatic problem (1.2)-(1.4), (1.6) is SbH. Unfortunately, we are not able to give an answer in the general case. In other words, the problem is $\mathrm{SbH}$ for (at least) the following models: (i) the viscoplastic model, exhibiting strain dependent viscosity $\nu^{\varepsilon}(x, \gamma)$, with non oscillating strain-rate sensitivity $n^{\star}(x)$ and with temperature independent non oscillating heat coefficient $c^{\star}(x)$; (ii) the thermoviscous model, exhibiting temperature dependent viscosity $\mu^{\varepsilon}(x, \theta)$, with non oscillating strain-rate sensitivity $n^{\star}(x)$ and with temperature dependent heat coefficient $c^{\varepsilon}(x, \theta)$; (iii) the general thermoviscoplastic model $\psi^{\varepsilon}(x, \gamma, \theta)$, with non oscillating strain-rate sensitivity $n^{\star}(x)$ and with temperature dependent heat coefficient $c^{\varepsilon}(x, \theta)$, in the case where the material is sheared by steady boundary shearing and body force. In the 
latest case, the homogenized viscosity $\psi^{0}$ and heat coefficient $c^{0}$ depend on the boundary shearing and of the body force. In all the three cases (i)-(iii), the homogenized coefficients depend on the initial values of the strain or of the temperature. This reflects the non elastic character of the problem (1.2)-(1.4), (1.6).

A particular, but of practical interest, remark is that we are unable to prove that the materials exhibiting an oscillating strain-rate sensitivity are $\mathrm{SbH}$ (see Remark 3.6). On the other hand, in the case of a (even smoothly) time dependent force and/or boundary shearing, the general thermoviscoplastic setting (with general coefficients $\psi^{\varepsilon}(x, \gamma, \theta)$ and $c^{\varepsilon}(x, \theta)$ ) is no more $\mathrm{SbH}$, since in general a memory effect appears in the viscosity coefficient (see Remark 5.5).

For the models (i) and (ii), the heterogeneous and the homogenized materials share the same type of constitutive laws, and these constitutive laws are independent of the boundary conditions. This fact provides an easy characterization of the mechanical behavior of the homogenized material and allows an easy exploitation of experimental data. For analogous reasons, the model (iii) may be useful for the prediction of effective properties, provided that the boundary shearing and the body force are steady.

The paper is organized as follows. In Section 2 we present an existence and uniqueness result for the general quasistatic problem and define the notion of stability by homogenization $(\mathrm{SbH})$ of the system describing the problem. In Sections 3 and 4 we prove that the system is $\mathrm{SbH}$ for two cases of materials: the case where the viscosity coefficient does not depend on the temperature (viscoplastic case) and the case where the viscosity coefficient does not depend on the strain (thermoviscous case). Finally, in Section 5, we prove that the general thermoviscoplastic material submitted to steady 
shearing and body force is SbH. In Sections 3 and 5, we also present numerical examples concerned with multiphase stratified materials with periodic structure when the phases are characterized by power laws; we observe that the homogenized materials are no more characterized by power laws, and therefore power laws are not $\mathrm{SbH}$.

\section{Existence and uniqueness result, and defi- nition of the Stability by Homogenization $(\mathrm{SbH})$}

In this Section we consider the general quasistatic problem posed in $Q=(0, T) \times \Omega$, where $T>0$ and where $\Omega=(a, b)$ is one-dimensional, namely

$$
\begin{gathered}
-\frac{\partial \sigma^{\varepsilon}}{\partial x}=f \quad \text { in } Q \\
\frac{\partial \gamma^{\varepsilon}}{\partial t}=\frac{\partial v^{\varepsilon}}{\partial x} \quad \text { in } Q \\
c^{\varepsilon}\left(x, \theta^{\varepsilon}\right) \frac{\partial \theta^{\varepsilon}}{\partial t}=\sigma^{\varepsilon} \frac{\partial v^{\varepsilon}}{\partial x} \quad \text { in } Q \\
\sigma^{\varepsilon}=\psi^{\varepsilon}\left(x, \gamma^{\varepsilon}, \theta^{\varepsilon}\right)\left|\frac{\partial v^{\varepsilon}}{\partial x}\right|^{n^{\varepsilon}(x)-1} \frac{\partial v^{\varepsilon}}{\partial x} \quad \text { in } Q
\end{gathered}
$$

complemented by the initial conditions

$$
\begin{aligned}
& \gamma^{\varepsilon}(0, x)=\gamma_{0}(x) \quad \text { in } \Omega, \\
& \theta^{\varepsilon}(0, x)=\theta_{0}(x) \quad \text { in } \Omega,
\end{aligned}
$$

and by the boundary conditions

$$
\begin{aligned}
& \sigma^{\varepsilon}(t, a)=\sigma_{a}(t) \quad \text { in }(0, T), \\
& v^{\varepsilon}(t, b)=v_{b}(t) \quad \text { in }(0, T) .
\end{aligned}
$$


In this system, the unknowns are the stress $\sigma^{\varepsilon}=\sigma^{\varepsilon}(t, x)$, the velocity $v^{\varepsilon}=v^{\varepsilon}(t, x)$, the strain $\gamma^{\varepsilon}=\gamma^{\varepsilon}(t, x)$ and the temperature $\theta^{\varepsilon}=\theta^{\varepsilon}(t, x)$. The first equation is the equilibrium equation, the second the compatibility equation, the third the energy equation and the fourth the constitutive law. The heat coefficient $c^{\varepsilon}\left(x, \theta^{\varepsilon}\right)$ is a temperature dependent function and the viscosity coefficient $\psi^{\varepsilon}\left(x, \gamma^{\varepsilon}, \theta^{\varepsilon}\right)$ is a strain and temperature dependent function. The force $f(t, x)$, the heat coefficient $c^{\varepsilon}(x, \theta)$, the viscosity coefficient $\psi^{\varepsilon}(x, \gamma, \theta)$, the strain-rate sensitivity $n^{\varepsilon}(x)$, the initial strain $\gamma_{0}(x)$, the initial temperature $\theta_{0}(x)$, the boundary stress $\sigma_{a}(t)$ and the boundary velocity $v_{b}(t)$ are data.

In this Section we first prove that under some conditions on the data, problem (2.1)-(2.8) has a unique solution (Proposition 2.1). We then define the notion of Stability by Homogenization $(\mathrm{SbH})$ of this system (Definition 2.2). Three particular cases where system (2.1)-(2.8) is $\mathrm{SbH}$ will be presented in Sections 3, 4 and 5 (see Proposition 3.1, Proposition 4.1 and Proposition $5.1)$.

Recall that in our papers Charalambakis and Murat (1989), Charalambakis and Murat (2006a) and Charalambakis and Murat (2006b) we considered the dynamical problem associated with (2.1)-(2.8) in the special case where $n^{\varepsilon}(x)=1$ (linear setting) and where $\psi^{\varepsilon}(x, \theta, \gamma)=\mu^{\varepsilon}(x, \theta)$ with $\frac{\partial \mu^{\varepsilon}}{\partial \theta}(x, \theta) \leq 0$ (thermoviscous problem with thermal softening). In this case we proved results of existence, uniqueness and stability by homogenization. Note that in that setting $\gamma^{\varepsilon}$ is a by-product of the problem in $\left(\sigma^{\varepsilon}, v^{\varepsilon}, \theta^{\varepsilon}\right)$, since once $v^{\varepsilon}$ is determined, $\gamma^{\varepsilon}$ is obtained by solving (2.2), (2.5), namely

$$
\begin{aligned}
& \frac{\partial \gamma^{\varepsilon}}{\partial t}=\frac{\partial v^{\varepsilon}}{\partial x} \quad \text { in } Q, \\
& \gamma^{\varepsilon}(0, x)=\gamma_{0}(x) \text { in } \Omega .
\end{aligned}
$$




\section{$2.1 \quad$ Existence and uniqueness}

We begin with an existence and uniqueness result in the general case. We make the following hypotheses on the data, where $0<M<+\infty$, $0<\alpha \leq \beta<+\infty$ and $0<\bar{\alpha} \leq \bar{\beta}<+\infty$ are given:

$$
\begin{gathered}
f=-\frac{\partial g}{\partial x} \quad \text { in } Q, \text { where } g \in C^{0}(\bar{Q}), \\
x \rightarrow c^{\varepsilon}(x, \theta) \text { is measurable on } \Omega, \forall \theta \in \mathbf{R}, \\
\left|\frac{\partial c^{\varepsilon}}{\partial \theta}(x, \theta)\right| \leq M, \quad \text { a.e. } x \in \Omega, \forall \theta \in \mathbf{R}, \\
\alpha \leq c^{\varepsilon}(x, \theta) \leq \beta, \quad \text { a.e. } x \in \Omega, \forall \theta \in \mathbf{R}, \\
x \rightarrow \psi^{\varepsilon}(x, \gamma, \theta) \text { is measurable on } \Omega, \quad \forall \gamma \in \mathbf{R}, \quad \forall \theta \in \mathbf{R}, \\
\mid \begin{array}{c}
\frac{\partial \psi^{\varepsilon}}{\partial \gamma}(x, \theta, \gamma) \mid \leq M, \quad \text { a.e. } x \in \Omega, \forall \gamma \in \mathbf{R}, \forall \theta \in \mathbf{R}, \\
\left|\frac{\partial \psi^{\varepsilon}}{\partial \theta}(x, \theta, \gamma)\right| \leq M, \quad \text { a.e. } x \in \Omega, \forall \gamma \in \mathbf{R}, \forall \theta \in \mathbf{R}, \\
\alpha \leq \psi^{\varepsilon}(x, \gamma, \theta) \leq \beta, \quad \text { a.e. } x \in \Omega, \forall \gamma \in \mathbf{R}, \forall \theta \in \mathbf{R}, \\
n^{\varepsilon} \in L^{\infty}(\Omega), \\
\bar{\alpha} \leq n^{\varepsilon}(x) \leq \bar{\beta}, \quad \text { a.e. } x \in \Omega, \\
\gamma_{0} \in L^{\infty}(\Omega), \\
\theta_{0} \in L^{\infty}(\Omega), \\
\sigma_{a} \in C^{0}([0, T]), \\
v_{b} \in L^{\infty}(0, T) .
\end{array}
\end{gathered}
$$

When considering their regularity with respect to $x$, the only hypothesis made on the heat coefficient $c^{\varepsilon}$, on the viscosity coefficient $\psi^{\varepsilon}$ and on the strain-rate sensitivity $n^{\varepsilon}$ is that they are bounded from below and from above 
by strictly positive constants. More specifically, they are allowed to exhibit discontinuities with respect to the spatial variable $x$. The same is valid for the initial strain $\gamma_{0}$ and the initial temperature $\theta_{0}$. It is also worth noticing that we do not make any softening or hardening hypothesis on the material behavior, since the viscosity coefficient $\psi^{\varepsilon}$ may have a negative or positive or non signed derivative with respect to strain and/or temperature.

Proposition 2.1 When hypotheses (2.9)-(2.22) hold true, there exists a unique solution $\left(\sigma^{\varepsilon}, v^{\varepsilon}, \gamma^{\varepsilon}, \theta^{\varepsilon}\right)$ of $(2.1)-(2.8)$, which satisfies

$$
\begin{gathered}
\sigma^{\varepsilon}=\sigma^{\star} \quad \text { in } Q, \\
v^{\varepsilon} \in L^{\infty}(Q), \quad \frac{\partial v^{\varepsilon}}{\partial x} \in L^{\infty}(Q), \\
\gamma^{\varepsilon} \in L^{\infty}(Q), \quad \frac{\partial \gamma^{\varepsilon}}{\partial t} \in L^{\infty}(Q), \\
\theta^{\varepsilon} \in L^{\infty}(Q), \quad \frac{\partial \theta^{\varepsilon}}{\partial t} \in L^{\infty}(Q),
\end{gathered}
$$

where $\sigma^{\star} \in C^{0}(\bar{Q})$ is given by

$$
\sigma^{\star}(t, x)=\sigma_{a}(t)+g(t, x)-g(t, a) \quad \text { in } \bar{Q} .
$$

Moreover $\sigma^{\varepsilon}, v^{\varepsilon}, \frac{\partial v^{\varepsilon}}{\partial x}, \gamma^{\varepsilon}, \frac{\partial \gamma^{\varepsilon}}{\partial t}, \theta^{\varepsilon}$ and $\frac{\partial \theta^{\varepsilon}}{\partial t}$ are bounded in $L^{\infty}(Q)$ independently of $\varepsilon$.

Proof of Proposition 2.1. Equation (2.1) and condition (2.9) imply that $\sigma^{\varepsilon}(t, x)-g(t, x)$ is independent of $x$, and therefore equal to its value for $x=a$, i.e.

$$
\sigma^{\varepsilon}(t, x)-g(t, x)=\sigma_{a}(t)-g(t, a) \text { in } Q
$$

This proves the existence and uniqueness of $\sigma^{\varepsilon}$, as well as (2.23) and (2.27). 
Then in view of (2.16), equation (2.4) combined with (2.23) is equivalent to

$$
\left|\frac{d v^{\varepsilon}}{d x}\right|^{n^{\varepsilon}(x)-1} \frac{d v^{\varepsilon}}{d x}=\frac{\sigma^{\star}}{\psi^{\varepsilon}\left(x, \gamma^{\varepsilon}, \theta^{\varepsilon}\right)} \quad \text { in } Q
$$

or to

$$
\frac{d v^{\varepsilon}}{d x}=\frac{\left|\sigma^{\star}\right|^{\frac{1}{n^{\varepsilon}(x)}}-1}{\sigma^{\star}} \quad \text { in } Q,
$$

which defines $\frac{d v^{\varepsilon}}{d x}$ in function of $\gamma^{\varepsilon}$ and $\theta^{\varepsilon}$. This proves the existence and uniqueness of $\frac{d v^{\varepsilon}}{d x}$ (and therefore of $v^{\varepsilon}$ in view of the boundary condition (2.8)) once the existence and uniqueness of $\gamma^{\varepsilon}$ and $\theta^{\varepsilon}$ are proved. Moreover in view of $(2.16),(2.18)$ and $\sigma^{\star} \in C^{0}(\bar{Q})$, equation $(2.28)$ implies that $\frac{d v^{\varepsilon}}{d x}$ belongs to $L^{\infty}(Q)$ and is bounded in this space independently of $\varepsilon$. Combined with the boundary condition (2.8) on $v^{\varepsilon}$ and hypothesis (2.22) on $v_{b}$, this implies the existence and uniqueness of $v^{\varepsilon}$ satisfying (2.24) and the boundedness of $v^{\varepsilon}$ and $\frac{\partial v^{\varepsilon}}{\partial x}$ in $L^{\infty}(Q)$ independently of $\varepsilon$ once the existence and uniqueness of $\gamma^{\varepsilon}$ and $\theta^{\varepsilon}$ are proved.

Let us finally prove the existence and uniqueness of $\gamma^{\varepsilon}$ and $\theta^{\varepsilon}$ satisfying (2.25) and (2.26), and the corresponding bounds in $L^{\infty}(Q)$ independent of $\varepsilon$. In view of the previous results, the problem (2.1)-(2.8) is equivalent to

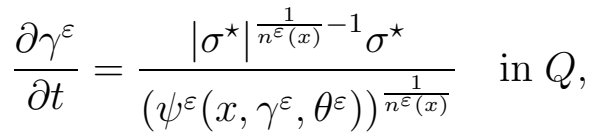

$$
\begin{aligned}
& \frac{\partial \theta^{\varepsilon}}{\partial t}=\frac{\left|\sigma^{\star}\right|^{\frac{1}{n^{\varepsilon}(x)}+1}}{c^{\varepsilon}\left(x, \theta^{\varepsilon}\right)\left(\psi^{\varepsilon}\left(x, \gamma^{\varepsilon}, \theta^{\varepsilon}\right)\right)^{\frac{1}{n^{\varepsilon}(x)}}} \text { in } Q, \\
& \gamma^{\varepsilon}(0, x)=\gamma_{0}(x) \text { in } \Omega \text {, } \\
& \theta^{\varepsilon}(0, x)=\theta_{0}(x) \quad \text { in } \Omega \text {. }
\end{aligned}
$$


For every $\varepsilon>0$ and a.e. $x \in \Omega$ fixed, problem (2.29)-(2.32) is an ordinary differential equation

$$
\begin{aligned}
& \frac{d X}{d t}=F(t, X) \quad \text { in }(0, T), \\
& X(0)=X_{0},
\end{aligned}
$$

where $X$ stands for the vector $\left(\gamma^{\varepsilon}, \theta^{\varepsilon}\right)$ and where $F(t, X)$ is continuous in $t$ (since $\sigma^{\star} \in C^{0}(\bar{Q})$ ) and Lipschitz continuous in $X$ (see (2.11), (2.12), (2.14), (2.15), (2.16) and (2.18)). Therefore this ordinary differential equation has a unique solution. This proves the existence and uniqueness of a solution of (2.29)-(2.32) (and therefore of $(2.1)-(2.8))$. In view of $\sigma^{\star} \in C^{0}(\bar{Q}),(2.12)$, (2.16), (2.18), (2.19) and (2.20), this unique solution satisfies (2.25), (2.26) and the corresponding estimates in $L^{\infty}(Q)$ independent of $\varepsilon$.

\subsection{Definition of the Stability by Homogenization ( $\mathrm{SbH}$ )}

Since we have $\sigma^{\varepsilon}=\sigma^{\star}$ for each $\varepsilon>0$ (see (2.23) in Proposition 2.1), we define $\sigma^{0}$ by

$$
\sigma^{0}=\sigma^{\star} \quad \text { in } Q
$$

and we have for every $\varepsilon>0$

$$
\sigma^{\varepsilon}=\sigma^{0} \quad \text { in } Q
$$

On the other hand, in view of the a priori bounds on $v^{\varepsilon}, \frac{\partial v^{\varepsilon}}{\partial x}, \gamma^{\varepsilon}, \frac{\partial \gamma^{\varepsilon}}{\partial t}, \theta^{\varepsilon}$ and $\frac{\partial \theta^{\varepsilon}}{\partial t}$ obtained in Proposition 2.1, it is possible to extract a subsequence $\varepsilon^{\prime}$ such that, as $\varepsilon^{\prime}$ tends to zero,

$$
v^{\varepsilon^{\prime}} \rightarrow v^{0} \quad \text { in } L^{\infty}(Q) \text { weak-star }
$$




$$
\begin{gathered}
\frac{\partial v^{\varepsilon^{\prime}}}{\partial x} \rightarrow \frac{\partial v^{0}}{\partial x} \quad \text { in } L^{\infty}(Q) \text { weak-star } \\
\gamma^{\varepsilon^{\prime}} \rightarrow \gamma^{0} \quad \text { in } L^{\infty}(Q) \text { weak-star, } \\
\frac{\partial \gamma^{\varepsilon^{\prime}}}{\partial t} \rightarrow \frac{\partial \gamma^{0}}{\partial t} \quad \text { in } L^{\infty}(Q) \text { weak-star }, \\
\theta^{\varepsilon^{\prime}} \rightarrow \theta^{0} \quad \text { in } L^{\infty}(Q) \text { weak-star }, \\
\frac{\partial \theta^{\varepsilon^{\prime}}}{\partial t} \rightarrow \frac{\partial \theta^{0}}{\partial t} \quad \text { in } L^{\infty}(Q) \text { weak-star }
\end{gathered}
$$

for some $v^{0}, \gamma^{0}$, and $\theta^{0}$ which belong to $L^{\infty}(Q)$, such that $\frac{\partial v^{0}}{\partial x}, \frac{\partial \gamma^{0}}{\partial t}$ and $\frac{\partial \theta^{0}}{\partial t}$ belong to $L^{\infty}(Q)$.

It is then easy to pass to the limit in the linear equations of problem (2.1)-(2.8), namely (2.1), (2.2), and (2.5)-(2.8), obtaining

$$
\begin{gathered}
-\frac{\partial \sigma^{0}}{\partial x}=f \quad \text { in } Q, \\
\frac{\partial \gamma^{0}}{\partial t}=\frac{\partial v^{0}}{\partial x} \quad \text { in } Q, \\
\gamma^{0}(0, x)=\gamma_{0}(x) \quad \text { in } \Omega, \\
\theta^{0}(0, x)=\theta_{0}(x) \quad \text { in } \Omega, \\
\sigma^{0}(t, a)=\sigma_{a}(t) \quad \text { in }(0, T), \\
v^{0}(t, b)=v_{b}(t) \quad \text { in }(0, T) .
\end{gathered}
$$

In contrast, it is not clear whether one can pass to the limit in the nonlinear equations (2.3) and (2.4), and obtain equations similar to (2.3) and (2.4), which would read as

$$
\begin{gathered}
c^{0}\left(x, \theta^{0}\right) \frac{\partial \theta^{0}}{\partial t}=\sigma^{0} \frac{\partial v^{0}}{\partial x} \quad \text { in } Q, \\
\sigma^{0}=\psi^{0}\left(x, \gamma^{0}, \theta^{0}\right)\left|\frac{\partial v^{0}}{\partial x}\right|^{n^{0}(x)-1} \frac{\partial v^{0}}{\partial x} \quad \text { in } Q,
\end{gathered}
$$


for some homogenized coefficients $c^{0}(x, \theta)$ and $\psi^{0}(x, \gamma, \theta)$ and some strainrate sensitivity $n^{0}(x)$.

This leads to the following definition.

Definition 2.2 Assume that hypotheses (2.9)-(2.22) hold true and let $\left(\sigma^{\varepsilon}, v^{\varepsilon}, \gamma^{\varepsilon}, \theta^{\varepsilon}\right)$ be the unique solution of problem (2.1)-(2.8). The problem (2.1)-(2.8) is said to be Stable by Homogenization (SbH) if there exists a subsequence $\varepsilon^{\prime}$, a heat coefficient $c^{0}(x, \theta)$, a viscosity coefficient $\psi^{0}(x, \gamma, \theta)$ and a strain-rate sensitivity $n^{0}(x)$ satisfying (2.10)-(2.18) (with constants possibly different of $M, \alpha, \beta, \bar{\alpha}$ and $\bar{\beta})$ such that for this subsequence (2.35)-(2.40) holds true for the unique solution $\left(\sigma^{0}, v^{0}, \gamma^{0}, \theta^{0}\right)$ of problem (2.41)-(2.48).

Then $c^{0}(x, \theta), \psi^{0}(x, \gamma, \theta)$ and $n^{0}(x)$ are called the homogenized heat coefficient, the homogenized viscosity coefficient and the homogenized strain-rate sensitivity of problem (2.1)-(2.8).

Note that in Definition 2.2 the homogenized quantities $c^{0}(x, \theta), \psi^{0}(x, \gamma, \theta)$ and $n^{0}(x)$ can depend on the force $f$ and on the initial and boundary data $\gamma_{0}$, $\theta_{0}, \sigma_{a}$ and $v_{b}$. This is a consequence of the non elastic character of problem $(2.1)-(2.8)$.

Unfortunately we are not able to prove that problem (2.1)-(2.8) is $\mathrm{SbH}$ when the general hypotheses (2.9)-(2.22) hold true. We are only able to deal with three special cases: in Sections 3,4 and 5 we will prove that when $n^{\varepsilon}(x)=n^{\star}(x)$ and when either

$$
\psi^{\varepsilon}(x, \gamma, \theta)=\nu^{\varepsilon}(x, \gamma) \quad \text { and } \quad c^{\varepsilon}(x, \theta)=c^{\star}(x),
$$

or when

$$
\psi^{\varepsilon}(x, \gamma, \theta)=\mu^{\varepsilon}(x, \gamma),
$$


or finally when

$$
\sigma^{\star}(t, x)=\sigma^{\star \star}(x)
$$

the system (2.1)-(2.8) is SbH (see Proposition 3.1, Proposition 4.1 and Proposition 5.1).

\section{Problem (2.1)-(2.8) is $\mathrm{SbH}$ when the vis- cosity coefficient $\psi^{\varepsilon}(x, \gamma, \theta)$ does not depend on the temperature $\theta$ (viscoplastic case)}

Proposition 3.1 Assume that hypotheses (2.9)-(2.22) hold true and moreover that

$$
\begin{gathered}
n^{\varepsilon}(x)=n^{\star}(x), \\
c^{\varepsilon}(x, \theta)=c^{\star}(x), \\
\psi^{\varepsilon}(x, \gamma, \theta)=\nu^{\varepsilon}(x, \gamma),
\end{gathered}
$$

for some given $n^{\star}(x), c^{\star}(x)$ and $\nu^{\varepsilon}(x, \gamma)$. Then problem (2.1)-(2.8) is $S b H$ and one has

$$
\begin{gathered}
n^{0}(x)=n^{\star}(x), \\
c^{0}(x, \theta)=c^{\star}(x), \\
\psi^{0}(x, \gamma, \theta)=\nu^{0}(x, \gamma),
\end{gathered}
$$

where the homogenized viscosity coefficient $\nu^{0}(x, \gamma)$ depends only on the sequence $\nu^{\varepsilon}(x, \gamma)$, on the strain-rate sensitivity $n^{\star}(x)$ and on the initial strain $\gamma^{0}(x)$. The homogenized viscosity coefficient $\nu^{0}(x, \gamma)$ is described in Remark 3.2 below. 
Proposition 3.1 asserts that when the strain-rate sensitivity $n^{\varepsilon}(x)$ is independent of $\varepsilon\left(n^{\varepsilon}(x)=n^{\star}(x)\right)$, when the heat coefficient $c^{\varepsilon}(x, \theta)$ is independent of $\varepsilon$ and of $\theta\left(c^{\varepsilon}(x, \theta)=c^{\star}(x)\right)$ and when the viscosity coefficient $\psi^{\varepsilon}(x, \gamma, \theta)$ is independent of $\theta\left(\psi^{\varepsilon}(x, \gamma, \theta)=\nu^{\varepsilon}(x, \gamma)\right)$, then problem $(2.1)-(2.8)$ is $\mathrm{SbH}$. Note that in this case the homogenized problem enjoys the same properties. In particular the homogenized viscosity coefficient is independent of $\theta$.

Proof of Proposition 3.1. Since here $c^{\varepsilon}(x, \theta)=c^{\star}(x)$ depends neither on $\varepsilon$ nor on $\theta$, it is straightforward to pass to the limit in equation (2.3), which reads as

$$
c^{\star}(x) \frac{\partial \theta^{\varepsilon}}{\partial t}=\sigma^{\star}(t, x) \frac{\partial v^{\varepsilon}}{\partial x} \quad \text { in } Q
$$

obtaining

$$
c^{\star}(x) \frac{\partial \theta^{0}}{\partial t}=\sigma^{\star}(t, x) \frac{\partial v^{0}}{\partial x} \quad \text { in } Q .
$$

The only problem is therefore to pass to the limit in the constitutive law (2.4), which thanks to (2.23) reads here as

$$
\sigma^{\star}(t, x)=\nu^{\varepsilon}\left(x, \gamma^{\varepsilon}\right)\left|\frac{\partial v^{\varepsilon}}{\partial x}\right|^{n^{\star}(x)-1} \frac{\partial v^{\varepsilon}}{\partial x} \quad \text { in } Q
$$

or equivalently as

$$
\frac{\partial v^{\varepsilon}}{\partial x}=\frac{\left|\sigma^{\star}(t, x)\right|^{\frac{1}{n^{\star}(x)}-1} \sigma^{\star}(t, x)}{\left(\nu^{\varepsilon}\left(x, \gamma^{\varepsilon}\right)\right)^{\frac{1}{n^{\star}(x)}}} \text { in } Q .
$$

By (2.2), (2.5) and (3.7) we therefore have

$$
\begin{aligned}
& \frac{\partial \gamma^{\varepsilon}}{\partial t}=\frac{\left|\sigma^{\star}(t, x)\right|^{\frac{1}{n^{\star}(x)}-1} \sigma^{\star}(t, x)}{\left(\nu^{\varepsilon}\left(x, \gamma^{\varepsilon}\right)\right)^{\frac{1}{n^{\star}(x)}}} \text { in } Q, \\
& \gamma^{\varepsilon}(0, x)=\gamma_{0}(x) \text { in } \Omega \text {. }
\end{aligned}
$$

Let us define the function $Z^{\varepsilon}: \Omega \times \mathbf{R} \rightarrow \mathbf{R}$ by

$$
Z^{\varepsilon}(x, s)=\int_{\gamma_{0}(x)}^{s}\left(\nu^{\varepsilon}\left(x, s^{\prime}\right)\right)^{\frac{1}{n^{\star}(x)}} d s^{\prime} \quad \text { in } \Omega \times \mathbf{R} .
$$


We have in particular

$$
\frac{\partial Z^{\varepsilon}}{\partial s}(x, s)=\left(\nu^{\varepsilon}(x, s)\right)^{\frac{1}{n^{\star}(x)}} \quad \text { in } \Omega \times \mathbf{R} .
$$

For a.e. $x \in \Omega$ fixed, the function $s \in \mathbf{R} \rightarrow Z^{\varepsilon}(x, s) \in \mathbf{R}$ is one-to-one, strictly increasing and Lipschitz continuous with

$$
0<\alpha^{1 / \bar{\beta}} \leq \frac{\partial Z^{\varepsilon}}{\partial s}(x, s) \leq \beta^{1 / \bar{\alpha}}<+\infty \quad \text { in } \Omega \times \mathbf{R},
$$

where $\alpha, \beta, \bar{\alpha}$ and $\bar{\beta}$ appear in (2.16) and (2.18). Therefore for a.e. $x \in \Omega$ fixed, this function has a reciprocal function $r \in \mathbf{R} \rightarrow\left(Z^{\varepsilon}\right)^{-1}(x, r) \in \mathbf{R}$ which is also one-to-one, strictly increasing and Lipschitz continuous with

$$
0<\frac{1}{\beta^{1 / \bar{\alpha}}} \leq \frac{\partial\left(Z^{\varepsilon}\right)^{-1}}{\partial r}(x, r) \leq \frac{1}{\alpha^{1 / \bar{\beta}}}<+\infty \quad \text { in } \Omega \times \mathbf{R}
$$

Then, since (3.8) reads as

$$
\frac{\partial Z^{\varepsilon}}{\partial s}\left(x, \gamma^{\varepsilon}\right) \frac{\partial \gamma^{\varepsilon}}{\partial t}=\left|\sigma^{\star}(t, x)\right|^{\frac{1}{n^{\star}(x)}-1} \sigma^{\star}(t, x) \text { in } Q
$$

and since

$$
Z^{\varepsilon}\left(x, \gamma_{0}(x)\right)=0 \quad \text { in } \Omega,
$$

we have

$$
Z^{\varepsilon}\left(x, \gamma^{\varepsilon}(t, x)\right)=K^{\star}(t, x) \text { in } Q
$$

where $K^{\star}=K^{\star}(t, x)$ is defined by

$$
\begin{aligned}
& \frac{\partial K^{\star}}{\partial t}=\left|\sigma^{\star}(t, x)\right|^{\frac{1}{n^{\star}(x)}-1} \sigma^{\star}(t, x) \text { in } Q, \\
& K^{\star}(0, x)=0 \quad \text { in } \Omega .
\end{aligned}
$$

Equation (3.13) is equivalent to

$$
\gamma^{\varepsilon}(t, x)=\left(Z^{\varepsilon}\right)^{-1}\left(x, K^{\star}(t, x)\right) \quad \text { in } Q .
$$


We finally define the function $\pi^{\varepsilon}: \Omega \times \mathbf{R} \rightarrow \mathbf{R}$ by

$$
\pi^{\varepsilon}(x, r)=\frac{1}{\left(\nu^{\varepsilon}\left(x,\left(Z^{\varepsilon}\right)^{-1}(x, r)\right)\right)^{\frac{1}{n^{\star}(x)}}} \text { in } \Omega \times \mathbf{R} .
$$

Observe that in view of (2.16) and (2.18), one has

$$
\frac{1}{\beta^{1 / \bar{\alpha}}} \leq \pi^{\varepsilon}(x, r) \leq \frac{1}{\alpha^{1 / \bar{\beta}}} \quad \text { in } \Omega \times \mathbf{R}
$$

In view of (2.14), (2.16), (2.18) and (3.12), we deduce from (3.16) that

$$
\begin{aligned}
& \left|\frac{\partial \pi^{\varepsilon}}{\partial r}(x, r)\right|= \\
& =\frac{1}{n^{\star}(x)} \frac{1}{\left(\nu^{\varepsilon}\left(x,\left(Z^{\varepsilon}\right)^{-1}(x, r)\right)\right)^{\frac{1}{n^{\star}(x)}+1}}\left|\frac{\partial \nu^{\varepsilon}}{\partial \gamma}\left(x,\left(Z^{\varepsilon}\right)^{-1}(x, r)\right)\right|\left|\frac{\partial\left(Z^{\varepsilon}\right)^{-1}}{\partial r}(x, r)\right| \leq \\
& \leq \frac{1}{\bar{\alpha}} \frac{1}{\alpha^{1+1 / \bar{\beta}}} M \frac{1}{\alpha^{1 / \bar{\beta}}} \quad \text { in } \Omega \times \mathbf{R}
\end{aligned}
$$

Since the functions $r \in \mathbf{R} \rightarrow\left(Z^{\varepsilon}\right)^{-1}(x, r) \in \mathbf{R}$ and $r \in \mathbf{R} \rightarrow \pi^{\varepsilon}(x, r) \in \mathbf{R}$ are (uniformly in $x$ and $\varepsilon$ ) Lipschitz continuous (see (3.12) and (3.17)), and since the functions $x \in \Omega \rightarrow\left(Z^{\varepsilon}\right)^{-1}(x, r) \in \mathbf{R}$ and $x \in \Omega \rightarrow \pi^{\varepsilon}(x, r) \in \mathbf{R}$ are measurable and bounded (uniformly in $\varepsilon$ ) for every $r \in \mathbf{R}$ fixed, a well known lemma in homogenization theory (see e.g. Lemma 3.8 in Charalambakis and Murat (2006b)) asserts that one can extract a subsequence $\varepsilon^{\prime}$ and that there exist two functions $Y^{0}(x, r)$ and $\pi^{0}(x, r): \Omega \times \mathbf{R} \rightarrow \mathbf{R}$ (which are also Lipschitz continuous in $r$ uniformly in $x$ and measurable in $x$ and bounded for every $r \in \mathbf{R}$ fixed), such that for every $r \in \mathbf{R}$ fixed

$$
\begin{gathered}
\left(Z^{\varepsilon^{\prime}}\right)^{-1}(x, r) \rightarrow Y^{0}(x, r) \text { in } L^{\infty}(\Omega) \text { weak-star }, \\
\pi^{\varepsilon^{\prime}}(x, r) \rightarrow \pi^{0}(x, r) \quad \text { in } L^{\infty}(\Omega) \text { weak-star. }
\end{gathered}
$$


Moreover the same lemma asserts that for this subsequence $\varepsilon^{\prime}$ one has

$$
\begin{gathered}
\left(Z^{\varepsilon^{\prime}}\right)^{-1}\left(x, K^{\star}(t, x)\right) \rightarrow Y^{0}\left(x, K^{\star}(t, x)\right) \text { in } L^{\infty}(Q) \text { weak-star, } \\
\pi^{\varepsilon^{\prime}}\left(x, K^{\star}(t, x)\right) \rightarrow \pi^{0}\left(x, K^{\star}(t, x)\right) \text { in } L^{\infty}(Q) \text { weak-star. }
\end{gathered}
$$

Since $Z^{\varepsilon}\left(x, \gamma^{0}(x)\right)=0$, we have

$$
\left(Z^{\varepsilon}\right)^{-1}(x, 0)=\gamma_{0}(x) \text { in } \Omega,
$$

and therefore

$$
Y^{0}(x, 0)=\gamma_{0}(x) \quad \text { in } \Omega .
$$

Since for every $r, r^{\prime} \in \mathbf{R}$ with $r \geq r^{\prime}$ we have (see (3.12))

$$
\frac{1}{\beta^{1 / \bar{\alpha}}}\left(r-r^{\prime}\right) \leq\left(Z^{\varepsilon}\right)^{-1}(x, r)-\left(Z^{\varepsilon}\right)^{-1}\left(x, r^{\prime}\right) \leq \frac{1}{\alpha^{1 / \bar{\beta}}}\left(r-r^{\prime}\right) \quad \text { in } \Omega,
$$

we also have for every $r, r^{\prime} \in \mathbf{R}$ with $r \geq r^{\prime}$

$$
\frac{1}{\beta^{1 / \bar{\alpha}}}\left(r-r^{\prime}\right) \leq Y^{0}(x, r)-Y^{0}\left(x, r^{\prime}\right) \leq \frac{1}{\alpha^{1 / \bar{\beta}}}\left(r-r^{\prime}\right) \quad \text { in } \Omega,
$$

which proves that for a.e. $x \in \Omega$ fixed, the function $r \in \mathbf{R} \rightarrow Y^{0}(x, r) \in \mathbf{R}$ is one-to-one, strongly increasing and Lipschitz continuous. Therefore this function has a reciprocal function $s \in \mathbf{R} \rightarrow\left(Y^{0}\right)^{-1}(x, s) \in \mathbf{R}$ with the same properties. We define the function $Z^{0}: \Omega \times \mathbf{R} \rightarrow \mathbf{R}$ by

$$
Z^{0}(x, s)=\left(Y^{0}\right)^{-1}(x, s) \text { in } \Omega \times \mathbf{R} .
$$

We also define the function $\nu^{0}: \Omega \times \mathbf{R} \rightarrow \mathbf{R}$ by

$$
\nu^{0}(x, s)=\frac{1}{\left(\pi^{0}\left(x, Z^{0}(x, s)\right)\right)^{n^{\star}(x)}} \quad \text { in } \Omega \times \mathbf{R},
$$

which is equivalent to (compare with (3.15))

$$
\pi^{0}(x, r)=\frac{1}{\left(\nu^{0}\left(x,\left(Z^{0}\right)^{-1}(x, r)\right)\right)^{\frac{1}{n^{\star}(x)}}} \text { in } \Omega \times \mathbf{R} .
$$


Since the function $\pi^{0}(x, r)$ is bounded from below and from above by strictly positive constants (this is easily deduced from (3.16) and (3.19)), the function $\nu^{0}(x, s)$ satisfies (2.16) (with constants possibly different of $\alpha$ and $\beta)$. On the other hand, since the function $Z^{0}(x, s)$ is Lipschitz continuous in $s$ uniformly in $x$ (see (3.22) and (3.23)) and since the function $\pi^{0}(x, r)$ is Lipschitz continuous in $s$ uniformly in $x$ (this can easily be deduced from (3.17) and (3.19)), the function $\nu^{0}(x, s)$ defined by (3.24) satisfies (2.14) (with a constant possibly different of $M$ ).

Then since by (3.14) one has $\gamma^{\varepsilon}(t, x)=\left(Z^{\varepsilon}\right)^{-1}\left(x, K^{\star}(t, x)\right)$, we deduce from (3.20) and (3.23) that

$$
\gamma^{\varepsilon^{\prime}} \rightarrow \gamma^{0} \quad \text { in } L^{\infty}(Q) \text { weak-star }
$$

where

$$
\gamma^{0}(t, x)=\left(Z^{0}\right)^{-1}\left(x, K^{\star}(t, x)\right) .
$$

On the other hand, we deduce from (3.14), (3.15), (3.21), (3.25) and (3.27) that

$$
\frac{1}{\left(\nu^{\varepsilon^{\prime}}\left(x, \gamma^{\varepsilon^{\prime}}(t, x)\right)\right)^{\frac{1}{n^{\star}(x)}}} \rightarrow \frac{1}{\left(\nu^{0}\left(x, \gamma^{0}(t, x)\right)\right)^{\frac{1}{n^{\star}(x)}}} \text { in } L^{\infty}(Q) \text { weak-star. }
$$

Turning back to (3.7), convergence (3.28) implies that

$$
\frac{\partial v^{0}}{\partial x}=\frac{\left|\sigma^{\star}(t, x)\right|^{\frac{1}{n^{\star}(x)}}-1}{\sigma^{\star}(t, x)} \quad \text { in } Q,
$$

which, thanks to (2.33), is equivalent to

$$
\sigma^{0}=\nu^{0}\left(x, \gamma^{0}\right)\left|\frac{\partial v^{0}}{\partial x}\right|^{n^{\star}(x)-1} \frac{\partial v^{0}}{\partial x} \quad \text { in } Q .
$$


In conclusion, we passed to the limit in (2.4) and therefore proved that in the setting of Proposition 3.1, the problem (2.1)-(2.8) is $\mathrm{SbH}$, with $n^{0}(x)=$ $n^{\star}(x), c^{0}(x, \theta)=c^{\star}(x)$ and $\psi^{0}(x, \gamma, \theta)=\nu^{0}(x, \gamma)$, with $\nu^{0}(x, \gamma)$ defined by (3.24). Note that the homogenized viscosity coefficient $\nu^{0}(x, \gamma)$ satisfies (2.13)-(2.16) (with constants possibly different of $M, \alpha$ and $\beta$ ).

Remark 3.2 Definition of the homogenized viscosity coefficient $\nu^{0}(x, \gamma)$

Let us summarize in this Remark the way in which $\nu^{0}(x, \gamma)$ is defined in the viscoplastic case considered in Proposition 3.1.

From the data $\nu^{\varepsilon}(x, s), n^{\star}(x)$ and $\gamma_{0}(x)$, we define the function $Z^{\varepsilon}(x, s)$ by

$$
Z^{\varepsilon}(x, s)=\int_{\gamma_{0}(x)}^{s}\left(\nu^{\varepsilon}\left(x, s^{\prime}\right)\right)^{\frac{1}{n^{\star}(x)}} d s^{\prime} \quad \text { in } \Omega \times \mathbf{R}
$$

(see (3.10)). Then we extract a subsequence $\varepsilon^{\prime}$ such that the reciprocal functions $\left(Z^{\varepsilon^{\prime}}\right)^{-1}(x, r)$ satisfy for every $r \in \mathbf{R}$ fixed

$$
\left(Z^{\varepsilon^{\prime}}\right)^{-1}(x, r) \rightarrow\left(Z^{0}\right)^{-1}(x, r) \quad \text { in } L^{\infty}(\Omega) \text { weak-star }
$$

for some function $Z^{0}(x, s)$ (see (3.18) and (3.23)). From the same data, we also define (see (3.15), (3.19) and (3.25)) a function $\nu^{0}(x, s)$ such that for every $r \in \mathbf{R}$ fixed (and possibly for a further subsequence, still denoted by $\left.\varepsilon^{\prime}\right)$ one has

$\frac{1}{\left(\nu^{\varepsilon^{\prime}}\left(x,\left(Z^{\varepsilon^{\prime}}\right)^{-1}(x, r)\right)\right)^{\frac{1}{n^{\star}(x)}}} \rightarrow \frac{1}{\left(\nu^{0}\left(x,\left(Z^{0}\right)^{-1}(x, r)\right)\right)^{\frac{1}{n^{\star}(x)}}}$ in $L^{\infty}(\Omega)$ weak-star.

This function $\nu^{0}(x, s)$ depends only (but does depend) on the sequence $\nu^{\varepsilon}(x, s)$, on the strain-rate sensitivity $n^{\star}(x)$ and on the initial condition $\gamma^{0}(x)$. It does not depend on the other data $\left(f, \theta_{0}, \sigma_{a}\right.$ and $\left.v_{b}\right)$ of the problem.

Then $\nu^{0}(x, \gamma)$ is the homogenized viscosity coefficient $\psi^{0}(x, \gamma, \theta)$ for which problem (2.1)-(2.8) is $\mathrm{SbH}$. 
Remark 3.3 The case of a multiphase viscoplastic heterogeneous material made of periodic homogeneous layers

In this Remark we consider the special case of Proposition 3.1 where the heterogeneous viscoplastic material is made of periodic thin layers (of thickness of order $\varepsilon$ ) of homogeneous phases.

In other words, we consider here the case where

$$
\begin{gathered}
n^{\star}(x)=n^{\star \star}, \\
c^{\star}(x)=c^{\star \star}, \\
\nu^{\varepsilon}(x, \gamma)=\sum_{i} \chi_{i}\left(\frac{x}{\varepsilon}\right) \nu_{i}(\gamma),
\end{gathered}
$$

where $n^{\star \star}$ and $c^{\star \star}$ are given in $\mathbf{R}^{+}$, where the index $i$ runs between 1 and $I$ ( $I \geq 2$ denotes the number of phases), where $\nu_{i}: \mathbf{R} \rightarrow \mathbf{R}$ are viscosity coefficients which do not depend on $x$ (and therefore describe homogeneous phases) and which satisfy (2.14) and (2.16), where

$$
0=a_{0}<a_{1}<\ldots<a_{i-1}<a_{I}=1
$$

are given numbers and where $\chi_{i}$ is the characteristic function of the interval $\left(a_{i-1}, a_{i}\right)$ extended by periodicity to $\mathbf{R}$, i.e.

$$
\chi_{i}(x)= \begin{cases}1 \quad \text { if } \quad k+a_{i-1}<x<k+a_{i} \quad \text { for some } k \in \mathbf{Z} \\ 0 \quad \text { otherwise. }\end{cases}
$$

We set

$$
p_{i}=a_{i}-a_{i-1}
$$

The number $p_{i}$ describes the volume fraction of the phase $i$ in the material and satisfies

$$
p_{i}>0 \quad \forall i, \quad \sum_{i} p_{i}=1 .
$$


Observe that

$$
\chi_{i}\left(\frac{x}{\varepsilon}\right) \rightarrow p_{i} \quad \text { in } L^{\infty}(\Omega) \text { weak-star. }
$$

We finally assume that

$$
\gamma_{0}(x)=\gamma^{\star \star} \quad \text { in } \Omega
$$

where $\gamma^{\star \star}$ is given in $\mathbf{R}$.

Hypotheses (2.9)-(2.22) are then satisfied, and the present setting is a particular case of Proposition 3.1. In this setting, the function $Z^{\varepsilon}$ defined by (3.10) is given by

$$
Z^{\varepsilon}(x, s)=\sum_{i} \chi_{i}\left(\frac{x}{\varepsilon}\right) \int_{\gamma^{\star \star}}^{s}\left(\nu_{i}\left(s^{\prime}\right)\right)^{\frac{1}{n^{\star \star}}} d s^{\prime}=\sum_{i} \chi_{i}\left(\frac{x}{\varepsilon}\right) N_{i}(s),
$$

where $N_{i}: \mathbf{R} \rightarrow \mathbf{R}$ is the function defined by

$$
N_{i}(s)=\int_{\gamma^{\star \star}}^{s}\left(\nu_{i}\left(s^{\prime}\right)\right)^{\frac{1}{n^{\star \star}}} d s^{\prime} .
$$

Therefore the reciprocal function $\left(Z^{\varepsilon}\right)^{-1}(x, r)$ is defined by

$$
\left(Z^{\varepsilon}\right)^{-1}(x, r)=\sum_{i} \chi_{i}\left(\frac{x}{\varepsilon}\right)\left(N_{i}\right)^{-1}(r)
$$

where $\left(N_{i}\right)^{-1}: \mathbf{R} \rightarrow \mathbf{R}$ is the reciprocal function of the function $N_{i}$.

In view of (3.36) the function $\left(Z^{0}\right)^{-1}(x, r)=Y^{0}(x, r)$ (which is defined by (3.18) and (3.23)) does not depend on $x$ and one has

$$
\left(Z^{0}\right)^{-1}(r)=Y^{0}(r)
$$

where

$$
Y^{0}(r)=\sum_{i} p_{i}\left(N_{i}\right)^{-1}(r)
$$

Similarly, the homogenized viscosity coefficient $\nu^{0}(x, s)$, which is defined by (3.15), (3.19) and (3.25), does not depend on $x$ : indeed, since the function 
$\pi^{\varepsilon}(x, r)$ defined by (3.15) is given here by

$$
\pi^{\varepsilon}(x, r)=\frac{1}{\left(\nu^{\varepsilon}\left(x,\left(Z^{\varepsilon}\right)^{-1}(x, r)\right)\right)^{\frac{1}{n^{\star}(x)}}}=\sum_{i} \frac{\chi_{i}\left(\frac{x}{\varepsilon}\right)}{\left(\nu_{i}\left(\left(N_{i}\right)^{-1}(r)\right)\right)^{\frac{1}{n^{\star}}}}
$$

we deduce from (3.19), (3.23), (3.25), (3.36) and (3.43) that

$$
\frac{1}{\left(\nu^{0}\left(Y^{0}(r)\right)\right)^{\frac{1}{n^{\star \star}}}}=\sum_{i} \frac{p_{i}}{\left(\nu_{i}\left(\left(N_{i}\right)^{-1}(r)\right)\right)^{\frac{1}{n^{\star \star}}}} .
$$

In the special setting of this Remark, which is concerned with periodic heterogeneous viscoplastic materials made of thin layers of homogeneous phases, equation (3.44) combined with (3.42) and (3.39) provides an explicit formula for the homogenized viscosity coefficient $\nu^{0}(x, \gamma)$.

Remark 3.4 The case of power laws for a multiphase viscoplastic heterogeneous material made of periodic layers

Let us complete the previous Remark by an explicit example.

In the setting of Remark 3.3, consider the case where the heterogeneous viscoplastic material is made of periodic layers of $I$ phases with volume fractions $p_{i}$, which are characterized by viscosity coefficients $\nu_{i}(s)$ which satisfy hypotheses (2.14) and (2.16) and which are given by power laws in some interval $A \leq s \leq B$ of $\mathbf{R}^{+}$, i.e. which satisfy

$$
\nu_{i}(s)=G_{i} s^{m_{i}}, \quad \forall s \quad \text { with } \quad A \leq s \leq B
$$

where $G_{i}$ are given in $\mathbf{R}^{+}, m_{i}$ are given in $\mathbf{R}$ and where $A$ and $B$ are given with $0<A<B<+\infty$. Note that viscosity coefficients defined by power laws on the whole of $\mathbf{R}$ or even of $\mathbf{R}^{+}$would satisfy neither hypothesis (2.14) nor hypothesis (2.16); this is the reason why we assume that the viscosity coefficients $\nu_{i}(s)$ of the phases are given by power laws only in the interval 
$A \leq s \leq B$. On the other hand, since we can have $A=\delta$ and $B=1 / \delta$ with $\delta>0$ small, the viscosity coefficients $\nu_{i}(s)$ can be defined as power laws on a very large part of $\mathbf{R}^{+}$.

We will assume that the powers $m_{i}$ and the initial strain $\gamma^{\star \star}$ satisfy

$$
\begin{gathered}
n^{\star \star}+m_{i} \neq 0, \\
\gamma^{\star \star} \leq A .
\end{gathered}
$$

These two hypotheses are only technical. Note that in (3.45) the power laws could be replaced by exponential or logarithmic laws (see Lemaitre and Chaboche (2001), Wright (2002)). Let us finally emphasize that the powers $m_{i}$ are assumed to be neither positive nor negative. This allows us to consider both softening and hardening processes, as well as problems with softening and hardening processes competing each other.

In this example the functions $N_{i}(s)$ defined by (3.39) are given by

$$
N_{i}(s)=g_{i} s^{\frac{n^{\star \star}+m_{i}}{n^{\star \star}}}-\kappa_{i}, \quad \forall s \quad \text { with } \quad A \leq s \leq B,
$$

(in the case where hypothesis (3.46) does not hold true, the power $\frac{n^{\star \star}+m_{i}}{n^{\star \star}}$ has to be replaced by a logarithm), where the constants $g_{i}$ and $\kappa_{i}$ are given by

$$
\begin{gathered}
g_{i}=\frac{n^{\star \star}}{n^{\star \star}+m_{i}} G_{i}^{\frac{1}{n^{\star \star}}}, \\
\kappa_{i}=g_{i} A^{\frac{n^{\star \star}+m_{i}}{n^{\star \star}}}-\int_{\gamma^{\star \star}}^{A}\left(\nu_{i}\left(s^{\prime}\right)\right)^{\frac{1}{n^{\star \star}}} d s^{\prime} .
\end{gathered}
$$

Note that

$$
\kappa_{i}=g_{i} A^{\frac{n^{\star \star}+m_{i}}{n^{\star \star}}} \text { when } A=\gamma^{\star \star} .
$$

The reciprocal function $\left(N_{i}\right)^{-1}(r)$ therefore satisfies

$$
\left(N_{i}\right)^{-1}(r)=\left(\frac{1}{g_{i}}\left(r+\kappa_{i}\right)\right)^{\frac{n^{\star \star}}{n^{\star \star}+m_{i}}}, \quad \forall r \quad \text { with } \quad N_{i}(A) \leq r \leq N_{i}(B) .
$$


Since the function $N_{i}(s)$ is strictly increasing and since $N_{i}\left(\gamma^{\star \star}\right)=0$, hypothesis (3.47) implies that $0 \leq N_{i}(A)<N_{i}(B)<+\infty$. We will moreover assume that there exist $C$ and $D$ such that

$$
\min _{i}\left(N_{i}(A)\right) \leq C<D \leq \max _{i}\left(N_{i}(B)\right) .
$$

Then on the interval $C \leq r \leq D$, the function $Y^{0}(r)$ defined by (3.42) is given by

$$
Y^{0}(r)=\sum_{i} p_{i}\left(\frac{1}{g_{i}}\left(r+\kappa_{i}\right)\right)^{\frac{n^{\star \star \star}}{n^{\star \star}+m_{i}}}, \quad \forall r \quad \text { with } \quad C \leq r \leq D,
$$

and formula (3.44) which defines the homogenized viscosity coefficient $\nu^{0}$ reads as

$$
\begin{gathered}
\frac{1}{\left(\nu^{0}\left(Y^{0}(r)\right)\right)^{\frac{1}{n^{\star \star}}}}=\sum_{i} \frac{p_{i}}{G_{i}^{\frac{1}{n^{\star \star}}}\left(\frac{1}{g_{i}}\left(r+\kappa_{i}\right)\right)^{\frac{m_{i}}{n^{\star \star}+m_{i}}}}, \\
\forall r \quad \text { with } \quad C \leq r \leq D .
\end{gathered}
$$

Formula (3.55) combined with (3.54) explicitly gives the value of the homogenized viscosity coefficient $\nu^{0}(s)$ in the interval $Y^{0}(C) \leq s \leq Y^{0}(D)$. Observe that the homogenized viscosity coefficient is no more given by a power law in this interval, which covers a very large part of $\mathbf{R}^{+}$when $A=\delta$ and $B=1 / \delta$ with $\delta>0$ small and when $\gamma^{\star \star}$ is close to zero. Therefore power laws are not $\mathrm{SbH}$.

\section{Remark 3.5 A numerical example}

We now present, in the context of Remark 3.4, some numerical results concerning the homogenized viscosity coefficient of a bimetallic material (so $I=2$ here) exhibiting strain hardening (so $m_{i}>0$ here) and strain-rate 
sensitivity $n^{\star \star}>0$. In this case the constitutive law (2.4) reads as

$$
\sigma=\sum_{i=1}^{2} \chi_{i}\left(\frac{x}{\varepsilon}\right) G_{i} \gamma^{m_{i}}\left|\frac{\partial v}{\partial x}\right|^{n^{\star \star}-1} \frac{\partial v}{\partial x}, \quad \forall \gamma \quad \text { with } \quad A \leq \gamma \leq B
$$

for some $A$ and $B$ given with $0<A<B<+\infty$ (see (3.45)). We moreover assume that the initial strain satisfies

$$
\gamma^{\star \star}=A
$$

Formulas (3.55) and (3.54) which define the homogenized viscosity coefficient $\nu^{0}$, together with (3.49) and (3.51), give

$$
\begin{gathered}
\frac{1}{\left(\nu^{0}\left(Y^{0}(r)\right)\right)^{\frac{1}{n^{\star \star}}}}=\sum_{i=1}^{2} \frac{p_{i}}{G_{i}^{\frac{1}{n^{\star \star}+m_{i}}}\left(\frac{n^{\star \star}+m_{i}}{n^{\star \star}} r+G_{i}^{\frac{1}{n^{\star}}}\left(\gamma^{\star \star}\right)^{\frac{n^{\star \star}+m_{i}}{n^{\star \star}}}\right)^{\frac{m_{i}}{n^{\star \star}+m_{i}}}}, \\
Y^{0}(r)=\sum_{i=1}^{2} \frac{p_{i}}{G_{i}^{\frac{1}{n^{\star \star}+m_{i}}}}\left(\frac{n^{\star \star}+m_{i}}{n^{\star \star}} r+G_{i}^{\frac{1}{n^{\star \star}}}\left(\gamma^{\star \star}\right)^{\frac{n^{\star \star}+m_{i}}{n^{\star \star}}}\right)^{\frac{n^{\star \star}}{n^{\star \star}+m_{i}}} \\
\forall r \quad \text { with } C \leq r \leq D .
\end{gathered}
$$

Lemaitre and Chaboche (2001) give the following range of values for the mechanical parameters in the constitutive law

$$
\begin{aligned}
& \text { constants } G_{1} \text { and } G_{2}: 100-10000 \mathrm{MPa} \text {, } \\
& \text { strain hardening } m_{1} \text { and } m_{2}: 0.02-0.5 \text {, } \\
& \text { strain rate-sensitivity } n^{\star \star}: 0.01-0.5 .
\end{aligned}
$$

We consider a layered material made of two equally distributed phases which are different steels ( so $p_{1}=p_{2}=\frac{1}{2}$ ), with

$$
G_{1}=762 \quad \mathrm{MPa}, \quad m_{1}=0.167, \quad n_{1}=0.07,
$$




$$
G_{2}=962 \quad \mathrm{MPa}, \quad m_{2}=0.187, \quad n_{2}=0.07,
$$

for different given values of the initial strain $\gamma^{\star \star}$.

Figures 1 and 2 present numerical results due to George Chatzigeorgiou, whose collaboration is gratefully acknowledged. Both figures present the values of the viscosity coefficients $\nu_{1}(r)$ and $\nu_{2}(r)$ given by the power law (3.56) with the numerical values given by (3.59) and (3.60), for the values $0.001,0.004,0.007$ and 0.010 of the initial strain $\gamma^{\star \star}$ (recall that we assumed that $A=\gamma^{\star \star}$, see (3.57)). Figures 1 and 2 also present the values of the homogenized viscosity coefficient $\nu^{0}(r)$ given by formula (3.58) for the values $0.001,0.004,0.007$ and 0.010 of the initial strain $\gamma^{\star \star}$. The two figures only differ by the range considered for $r$. In Figure 1, $r$ takes its values between 0 and $5 e+038=5.10^{38}$, while in Figure 2, $r$ takes its values between 0 and $4 e+035=4.10^{35}$ (the values of the variable in the horizontal axis have to be read according to the rule $k e+l=k 10^{l}$ ). Figure 1 shows that, for large values of $r$, the homogenized viscosity coefficient does not depend in practice on the initial value $\gamma^{\star \star}$, since the 4 curves seem to coincide. In contrast, for small values of $r$ (onset of the deformation), the homogenized viscosity coefficient depends on the initial value $\gamma^{\star \star}$ since the curves clearly differ. Finally, it is worth noticing that the homogenized viscosity coefficient is more "attracted" by the hardening of the weaker material than by the hardening of the stronger one, in a ratio $2 / 1$.

Remark 3.6 A comment on the hypothesis $c^{\varepsilon}(x, \theta)=c^{\star}(x)$

In Proposition 3.1, the heat coefficient $c^{\varepsilon}(x, \theta)$ is assumed to be independent of $\varepsilon$ and of $x$ (see hypothesis (3.2)). One can wonder whether this hypothesis is necessary or not, i.e. whether one could pass to the limit in equation (2.3) when the heat coefficient is of the general form $c^{\varepsilon}(x, \theta)$. 
Thanks to (2.23), equation (2.3) reads as

$$
c^{\varepsilon}\left(x, \theta^{\varepsilon}\right) \frac{\partial \theta^{\varepsilon}}{\partial t}=\sigma^{\star} \frac{\partial v^{\varepsilon}}{\partial x} \quad \text { in } Q .
$$

Defining the function $C^{\varepsilon}: \Omega \times \mathbf{R} \rightarrow \mathbf{R}$ by

$$
C^{\varepsilon}(x, s)=\int_{\theta_{0}(x)}^{s} c^{\varepsilon}\left(x, s^{\prime}\right) d s^{\prime} \quad \text { in } \Omega \times \mathbf{R},
$$

and defining the transformed temperature $\tau^{\varepsilon}=\tau^{\varepsilon}(t, x)$ by

$$
\tau^{\varepsilon}(t, x)=C^{\varepsilon}\left(x, \theta^{\varepsilon}(t, x)\right) \quad \text { in } Q
$$

equation (3.61) reads as

$$
\frac{\partial \tau^{\varepsilon}}{\partial t}=\sigma^{\star} \frac{\partial v^{\varepsilon}}{\partial x} \text { in } Q
$$

while the initial condition $(2.6)$ on $\theta^{\varepsilon}$ yields

$$
\tau^{\varepsilon}(0, x)=0 \quad \text { in } \Omega
$$

Combining (3.63) and (3.64) with the convergence (2.36) implies that

$$
\begin{gathered}
\tau^{\varepsilon^{\prime}} \rightarrow \tau^{0} \quad \text { in } L^{\infty}(Q) \text { weak-star } \\
\frac{\partial \tau^{\varepsilon^{\prime}}}{\partial t} \rightarrow \frac{\partial \tau^{0}}{\partial t} \quad \text { in } L^{\infty}(Q) \text { weak-star }
\end{gathered}
$$

where $\tau^{0}$ is the solution of

$$
\begin{gathered}
\frac{\partial \tau^{0}}{\partial t}=\sigma^{\star} \frac{\partial v^{0}}{\partial x} \quad \text { in } Q \\
\tau^{0}(0, x)=0 \quad \text { in } \Omega,
\end{gathered}
$$

which defines in a unique way the limit $\tau^{0}$ of the transformed temperature $\tau^{\varepsilon^{\prime}}$ 
But since $\tau^{\varepsilon^{\prime}}$ and $\theta^{\varepsilon^{\prime}}$ converge only weakly, one cannot pass to the limit in the relation (3.62) and deduce a relation between $\tau^{0}$ defined by (3.65)-(3.66) and $\theta^{0}$ defined by (2.39). This is impossible even in the case where $c^{\varepsilon}(x, \theta)=c^{\varepsilon}(x)$ does not depend on $\theta$. Indeed, in this case, (3.62) reads as

$$
\tau^{\varepsilon}(t, x)=c^{\varepsilon}(x)\left(\theta^{\varepsilon}(t, x)-\theta_{0}(x)\right)
$$

in which we do not know how to pass to the limit in the product $c^{\varepsilon}(x) \theta^{\varepsilon}(t, x)$ (we would not know how to pass to the limit in this product even if we knew that $\theta^{\varepsilon}$ does not depend on $t$ ). This is the reason which forces us to assume that $c^{\varepsilon}(x, \theta)$ is independent of $\theta$ and of $\varepsilon$ (hypothesis 3.2)).

The same reason forces us to assume in Proposition 3.1 that the strainrate sensitivity $n^{\varepsilon}$ does not depend on $\varepsilon$ (hypothesis (3.1)).

In contrast, the key point in the proof of Proposition 3.1 is the fact that one has

$$
\gamma^{\varepsilon}(t, x)=\left(Z^{\varepsilon}\right)^{-1}\left(x, K^{\star}(t, x)\right) \quad \text { in } Q
$$

(see $(3.14))$, i.e. the fact that $\gamma^{\varepsilon}$ is a function of the fixed function $K^{\star}$. This fact allows us to obtain a relation between the limit $\gamma^{0}$ of $\gamma^{\varepsilon^{\prime}}$ and the function $K^{\star}$, and makes the success of the above proof.

\section{Problem (2.1)-(2.8) is $\mathrm{SbH}$ when the vis- cosity coefficient $\psi^{\varepsilon}(x, \gamma, \theta)$ does not depend on the strain $\gamma$ (thermoviscous case)}

Proposition 4.1 Assume that hypotheses (2.9)-(2.22) hold true and moreover that

$$
n^{\varepsilon}(x)=n^{\star}(x)
$$




$$
\psi^{\varepsilon}(x, \gamma, \theta)=\mu^{\varepsilon}(x, \theta),
$$

for some given $n^{\star}(x)$ and $\mu^{\varepsilon}(x, \theta)$. Then problem (2.1)-(2.8) is SbH and one has

$$
\begin{gathered}
n^{0}(x)=n^{\star}(x), \\
\psi^{0}(x, \gamma, \theta)=\mu^{0}(x, \theta),
\end{gathered}
$$

where the homogenized heat coefficient $c^{0}(x, \theta)$ and the homogenized viscosity coefficient $\mu^{0}(x, \theta)$ depend only on the sequences $c^{\varepsilon}(x, \theta)$ and $\mu^{\varepsilon}(x, \theta)$, on the strain-rate sensitivity $n^{\star}(x)$ and on the initial temperature $\theta^{0}(x)$. The homogenized coefficients $c^{0}(x, \theta)$ and $\mu^{0}(x, \theta)$ are described in Remark 4.2 below.

Proposition 4.1 asserts that when the strain-rate sensitivity $n^{\varepsilon}(x)$ is independent of $\varepsilon\left(n^{\varepsilon}(x)=n^{\star}(x)\right)$ and when the viscosity coefficient $\psi^{\varepsilon}(x, \gamma, \theta)$ is independent of $\gamma\left(\psi^{\varepsilon}(x, \gamma, \theta)=\mu^{\varepsilon}(x, \theta)\right)$, then problem (2.1)-(2.8) is $\mathrm{SbH}$. Note that in this case the homogenized problem enjoys the same properties. In particular the homogenized viscosity coefficient is independent of $\gamma$.

The result of Proposition 4.1 is close to the homogenization result obtained in Charalambakis and Murat (2006b) which is concerned with the dynamical problem associated to $(2.1)-(2.8)$ in the case where $\psi^{\varepsilon}(x, \gamma, \theta)=$ $=\mu^{\varepsilon}(x, \theta)$. Observe however that the result of Charalambakis and Murat (2006b) is obtained under the stronger hypotheses that $n^{\star}(x)=1$ (linear setting) and $\frac{\partial \mu^{\varepsilon}}{\partial \theta}(x, \theta) \leq 0$ (thermal softening).

Proof of Proposition 4.1. The proof is very similar to the proof of Proposition 3.1. The problem is to pass to the limit in (2.3) and (2.4).

In view of (2.23), equation (2.4) is here equivalent to

$$
\frac{\partial v^{\varepsilon}}{\partial x}=\frac{\left|\sigma^{\star}(t, x)\right|^{\frac{1}{n^{\star}(x)}}-1 \sigma^{\star}(t, x)}{\left(\mu^{\varepsilon}\left(x, \theta^{\varepsilon}\right)\right)^{\frac{1}{n^{\star}(x)}}} \text { in } Q,
$$


and therefore (2.3) reads as

$$
c^{\varepsilon}\left(x, \theta^{\varepsilon}\right) \frac{\partial \theta^{\varepsilon}}{\partial t}=\frac{\left|\sigma^{\star}(t, x)\right|^{\frac{1}{n^{\star}(x)}+1}}{\left(\mu^{\varepsilon}\left(x, \theta^{\varepsilon}\right)\right)^{\frac{1}{n^{\star}(x)}}} \text { in } Q,
$$

or equivalently as

$$
c^{\varepsilon}\left(x, \theta^{\varepsilon}\right)\left(\mu^{\varepsilon}\left(x, \theta^{\varepsilon}\right)\right)^{\frac{1}{n^{\star}(x)}} \frac{\partial \theta^{\varepsilon}}{\partial t}=\left|\sigma^{\star}(t, x)\right|^{\frac{1}{n^{\star}(x)}+1} \quad \text { in } Q .
$$

Let us define the function $\hat{Z}^{\varepsilon}: \Omega \times \mathbf{R} \rightarrow \mathbf{R}$ by

$$
\hat{Z}^{\varepsilon}(x, s)=\int_{\theta_{0}(x)}^{s} c^{\varepsilon}\left(x, s^{\prime}\right)\left(\mu^{\varepsilon}\left(x, s^{\prime}\right)\right)^{\frac{1}{n^{\star}(x)}} d s^{\prime} \quad \text { in } \Omega \times \mathbf{R} .
$$

We have in particular

$$
\frac{\partial \hat{Z}^{\varepsilon}}{\partial s}(x, s)=c^{\varepsilon}\left(x, s^{\prime}\right)\left(\mu^{\varepsilon}(x, s)\right)^{\frac{1}{n^{\star}(x)}} \quad \text { in } \Omega \times \mathbf{R} .
$$

For a.e. $x \in \Omega$ fixed, the function $s \in \mathbf{R} \rightarrow \hat{Z}^{\varepsilon}(x, s) \in \mathbf{R}$ is one-to-one, strictly increasing and Lipschitz continuous with

$$
0<\alpha^{1+1 / \bar{\beta}} \leq \frac{\partial \hat{Z}^{\varepsilon}}{\partial s}(x, s) \leq \beta^{1+1 / \bar{\alpha}}<+\infty \quad \text { in } \Omega \times \mathbf{R},
$$

where $\alpha, \beta, \bar{\alpha}$ and $\bar{\beta}$ appear in (2.12), (2.16) and (2.18). Therefore for a.e. $x \in \Omega$ fixed, this function has a reciprocal function $r \in \mathbf{R} \rightarrow\left(\hat{Z}^{\varepsilon}\right)^{-1}(x, r) \in \mathbf{R}$ which is also one-to-one, strictly increasing and Lipschitz continuous with

$$
0<\frac{1}{\beta^{1+1 / \alpha}} \leq \frac{\partial\left(\hat{Z}^{\varepsilon}\right)^{-1}}{\partial r}(x, r) \leq \frac{1}{\alpha^{1+1 / \bar{\beta}}}<+\infty \quad \text { in } \Omega \times \mathbf{R}
$$

Then, since (4.6) reads as

$$
\frac{\partial \hat{Z}^{\varepsilon}}{\partial s}\left(x, \theta^{\varepsilon}\right) \frac{\partial \theta^{\varepsilon}}{\partial t}=\left|\sigma^{\star}\right|^{\frac{1}{n^{\star}(x)}+1} \quad \text { in } Q
$$

and since

$$
\hat{Z}^{\varepsilon}\left(x, \theta_{0}(x)\right)=0 \quad \text { in } \Omega,
$$


we have

$$
\hat{Z}^{\varepsilon}\left(x, \theta^{\varepsilon}(t, x)\right)=H^{\star}(t, x) \quad \text { in } Q,
$$

where $H^{\star}=H^{\star}(t, x)$ is defined by

$$
\begin{aligned}
& \frac{\partial H^{\star}}{\partial t}=\left|\sigma^{\star}\right|^{\frac{1}{n^{\star}(x)}+1} \quad \text { in } Q, \\
& H^{\star}(0, x)=0 \quad \text { in } \Omega .
\end{aligned}
$$

Equation (4.10) is equivalent to

$$
\theta^{\varepsilon}(t, x)=\left(\hat{Z}^{\varepsilon}\right)^{-1}\left(x, H^{\star}(t, x)\right) \quad \text { in } Q .
$$

We finally define the function $\zeta^{\varepsilon}: \Omega \times \mathbf{R} \rightarrow \mathbf{R}$ by

$$
\zeta^{\varepsilon}(x, r)=\frac{1}{\left(\mu^{\varepsilon}\left(x,\left(\hat{Z}^{\varepsilon}\right)^{-1}(x, r)\right)\right)^{\frac{1}{n^{\star}(x)}}} \text { in } \Omega \times \mathbf{R} .
$$

Observe that in view of (2.16) and (2.18), one has

$$
\frac{1}{\beta^{1 / \bar{\alpha}}} \leq \zeta^{\varepsilon}(x, r) \leq \frac{1}{\alpha^{1 / \bar{\beta}}} \quad \text { in } \Omega \times \mathbf{R} .
$$

In view of (2.15), (2.16), (2.18) and (4.9), we deduce from (4.14) that

$$
\begin{aligned}
& \left|\frac{\partial \zeta^{\varepsilon}}{\partial r}(x, r)\right|= \\
& =\frac{1}{n^{\star}(x)} \frac{1}{\left(\mu^{\varepsilon}\left(x,\left(\hat{Z}^{\varepsilon}\right)^{-1}(x, r)\right)\right)^{\frac{1}{n^{\star}(x)}+1}}\left|\frac{\partial \mu^{\varepsilon}}{\partial \theta}\left(x,\left(\hat{Z}^{\varepsilon}\right)^{-1}(x, r)\right)\right|\left|\frac{\partial\left(\hat{Z}^{\varepsilon}\right)^{-1}}{\partial r}(x, r)\right| \leq \\
& \leq \frac{1}{\bar{\alpha}} \frac{1}{\alpha^{1+1 / \bar{\beta}}} M \frac{1}{\alpha^{1+1 / \bar{\beta}}} \quad \text { in } \Omega \times \mathbf{R} .
\end{aligned}
$$

Since the functions $r \in \mathbf{R} \rightarrow\left(\hat{Z}^{\varepsilon}\right)^{-1}(x, r) \in \mathbf{R}$ and $r \in \mathbf{R} \rightarrow \zeta^{\varepsilon}(x, r) \in \mathbf{R}$ are (uniformly in $x$ and $\varepsilon$ ) Lipschitz continuous (see (4.9) and (4.16)), and since the functions $x \in \Omega \rightarrow\left(\hat{Z}^{\varepsilon}\right)^{-1}(x, r) \in \mathbf{R}$ and $x \in \Omega \rightarrow \zeta^{\varepsilon}(x, r) \in \mathbf{R}$ are 
measurable and bounded (uniformly in $\varepsilon$ ) for every $r \in \mathbf{R}$ fixed, a well known lemma in homogenization theory (see e.g. Lemma 3.8 in Charalambakis and Murat (2006b)) asserts that one can extract a subsequence $\varepsilon^{\prime}$ and that there exist two functions $\hat{Y}^{0}(x, r)$ and $\zeta^{0}(x, r): \Omega \times \mathbf{R} \rightarrow \mathbf{R}$ (which are also Lipschitz continuous in $r$ uniformly in $x$ and measurable in $x$ and bounded for every $r \in \mathbf{R}$ fixed), such that for every $r \in \mathbf{R}$ fixed

$$
\begin{gathered}
\left(\hat{Z}^{\varepsilon^{\prime}}\right)^{-1}(x, r) \rightarrow \hat{Y}^{0}(x, r) \quad \text { in } L^{\infty}(\Omega) \text { weak-star, } \\
\zeta^{\varepsilon^{\prime}}(x, r) \rightarrow \zeta^{0}(x, r) \quad \text { in } L^{\infty}(\Omega) \text { weak-star. }
\end{gathered}
$$

Moreover the same lemma asserts that for this subsequence $\varepsilon^{\prime}$ one has

$$
\begin{gathered}
\left(\hat{Z}^{\varepsilon^{\prime}}\right)^{-1}\left(x, H^{\star}(t, x)\right) \rightarrow \hat{Y}^{0}\left(x, H^{\star}(t, x)\right) \text { in } L^{\infty}(Q) \text { weak-star, } \\
\zeta^{\varepsilon^{\prime}}\left(x, H^{\star}(t, x)\right) \rightarrow \zeta^{0}\left(x, H^{\star}(t, x)\right) \text { in } L^{\infty}(Q) \text { weak-star. }
\end{gathered}
$$

Since $\hat{Z}^{\varepsilon}\left(x, \theta^{0}(x)\right)=0$, we have

$$
\left(\hat{Z}^{\varepsilon}\right)^{-1}(x, 0)=\theta_{0}(x) \text { in } \Omega,
$$

and therefore

$$
\hat{Y}^{0}(x, 0)=\theta_{0}(x) \text { in } \Omega .
$$

Since for every $r, r^{\prime} \in \mathbf{R}$ with $r \geq r^{\prime}$ we have (see (4.9))

$$
\frac{1}{\beta^{1+1 / \bar{\alpha}}}\left(r-r^{\prime}\right) \leq\left(\hat{Z}^{\varepsilon}\right)^{-1}(x, r)-\left(\hat{Z}^{\varepsilon}\right)^{-1}\left(x, r^{\prime}\right) \leq \frac{1}{\alpha^{1+1 / \bar{\beta}}}\left(r-r^{\prime}\right) \quad \text { in } \Omega,
$$

we also have for every $r, r^{\prime} \in \mathbf{R}$ with $r \geq r^{\prime}$

$$
\frac{1}{\beta^{1+1 / \bar{\alpha}}}\left(r-r^{\prime}\right) \leq \hat{Y}^{0}(x, r)-\hat{Y}^{0}\left(x, r^{\prime}\right) \leq \frac{1}{\alpha^{1+1 / \bar{\beta}}}\left(r-r^{\prime}\right) \quad \text { in } \Omega,
$$

which proves that for a.e. $x \in \Omega$ fixed, the function $r \in \mathbf{R} \rightarrow \hat{Y}^{0}(x, r) \in \mathbf{R}$ is one-to-one, strongly increasing and Lipschitz continuous. Therefore this 
function has a reciprocal function $s \in \mathbf{R} \rightarrow\left(\hat{Y}^{0}\right)^{-1}(x, s) \in \mathbf{R}$ with the same properties. We define the function $\hat{Z}^{0}: \Omega \times \mathbf{R} \rightarrow \mathbf{R}$ by

$$
\hat{Z}^{0}(x, s)=\left(\hat{Y}^{0}\right)^{-1}(x, s) \quad \text { in } \Omega \times \mathbf{R} .
$$

We also define the function $\mu^{0}: \Omega \times \mathbf{R} \rightarrow \mathbf{R}$ by

$$
\mu^{0}(x, s)=\frac{1}{\left(\zeta^{0}\left(x, \hat{Z}^{0}(x, s)\right)\right)^{n^{\star}(x)}} \quad \text { in } \Omega \times \mathbf{R},
$$

which is equivalent to (compare with (4.14))

$$
\zeta^{0}(x, r)=\frac{1}{\left(\mu^{0}\left(x,\left(\hat{Z}^{0}\right)^{-1}(x, r)\right)\right)^{\frac{1}{n^{\star}(x)}}} \quad \text { in } \Omega \times \mathbf{R} .
$$

Since the function $\zeta^{0}(x, r)$ is bounded from below and from above by strictly positive constants (this is easily deduced from (4.15) and (4.18)), the function $\mu^{0}(x, s)$ satisfies (2.16) (with constants possibly different of $\alpha$ and $\beta$ ). On the other hand, since the function $\hat{Z}^{0}(x, s)$ is Lipschitz continuous in $s$ uniformly in $x$ (see (4.21) and (4.22)) and since the function $\zeta^{0}(x, r)$ is Lipschitz continuous in $r$ uniformly in $x$ (this can easily be deduced from (4.16) and (4.18)), the function $\mu^{0}(x, s)$ defined by (4.23) satisfies (2.15) (with a constant possibly different of $M$ ).

Then since by (4.13) $\theta^{\varepsilon}(t, x)=\left(\hat{Z}^{\varepsilon}\right)^{-1}\left(x, H^{\star}(t, x)\right)$, we deduce from (4.19) and (4.22) that

$$
\theta^{\varepsilon^{\prime}} \rightarrow \theta^{0} \quad \text { in } L^{\infty}(Q) \text { weak-star }
$$

where

$$
\theta^{0}(t, x)=\left(\hat{Z}^{0}\right)^{-1}\left(x, H^{\star}(t, x)\right)
$$

On the other hand, we deduce from (4.13), (4.14), (4.20), (4.24) and (4.26) that

$$
\frac{1}{\left(\mu^{\varepsilon^{\prime}}\left(x, \theta^{\varepsilon^{\prime}}(t, x)\right)\right)^{\frac{1}{n^{\star}(x)}}} \rightarrow \frac{1}{\left(\mu^{0}\left(x, \theta^{0}(t, x)\right)\right)^{\frac{1}{n^{\star}(x)}}} \text { in } L^{\infty}(Q) \text { weak-star. }
$$


Turning back to (4.5), convergence (4.27) implies that

$$
\frac{\partial v^{0}}{\partial x}=\frac{\left|\sigma^{\star}(t, x)\right|^{\frac{1}{n^{\star}(x)}-1} \sigma^{\star}(t, x)}{\left(\mu^{0}\left(x, \theta^{0}(t, x)\right)\right)^{\frac{1}{n^{\star}(x)}}} \text { in } Q,
$$

which, thanks to $(2.33)$, is equivalent to

$$
\sigma^{0}=\mu^{0}\left(x, \theta^{0}\right)\left|\frac{\partial v^{0}}{\partial x}\right|^{n^{\star}(x)-1} \frac{\partial v^{0}}{\partial x} \quad \text { in } Q .
$$

We passed to the limit in (2.4). Let us now pass to the limit in (2.3).

From the function $\hat{Z}^{0}$ defined by (4.17) and (4.22), and from the function $\mu^{0}$ defined by (4.23), we define the function $c^{0}: \Omega \times \mathbf{R} \rightarrow \mathbf{R}$ by

$$
c^{0}(x, s)=\frac{\frac{\partial \hat{Z}^{0}}{\partial s}(x, s)}{\left(\mu^{0}(x, s)\right)^{\frac{1}{n^{\star}(x)}}} \quad \text { in } \Omega \times \mathbf{R},
$$

or equivalently by

$$
\frac{\partial \hat{Z}^{0}}{\partial s}(x, s)=c^{0}(x, s)\left(\mu^{0}(x, s)\right)^{\frac{1}{n^{\star}(x)}} \quad \text { in } \Omega \times \mathbf{R}
$$

(compare with (4.8)). Since the functions $\frac{\partial \hat{Z}^{0}}{\partial s}(x, s)$ and $\mu^{0}(x, s)$ are bounded from below and from above by strictly positive constants, the function $c^{0}(x, s)$ is correctly defined by (4.29) and satisfies (2.12) (with constants possibly different of $\alpha$ and $\beta)$. Moreover, the function $c^{0}(x, s)$ defined by (4.29) satisfies (2.11) (with a constant possibly different of $M$ ): indeed we proved that the function $\mu^{0}(x, s)$ is Lipschitz continuous in $s$, and it can be proved that the function $\frac{\partial \hat{Z}^{0}}{\partial s}(x, s)$ is Lipschitz continuous in $s$ uniformly in $x$ : this is due to the fact that $\frac{\partial \hat{Z}^{\varepsilon}}{\partial s}(x, s)$ is Lipschitz continuous in $s$ uniformly in $x$ and $\varepsilon$ (see (4.8), (2.11) and (2.15)), and can be proved by a proof similar to the proof used to prove the similar property for the function $M^{0}(x, s)$ defined in Charalambakis and Murat (2006b) (see the proof of (3.45) in this paper). 
On the other hand, (4.11) and (4.26) imply that

$$
\frac{\partial \hat{Z}^{0}\left(x, \theta^{0}(t, x)\right)}{\partial t}=\left|\sigma^{\star}\right|^{\frac{1}{n^{\star}(x)}}+1 \quad \text { in } Q
$$

Combining (4.30) and (4.31) yields

$$
c^{0}\left(x, \theta^{0}\right)\left(\mu^{0}\left(x, \theta^{0}\right)\right)^{\frac{1}{n^{\star}(x)}} \frac{\partial \theta^{0}}{\partial t}=\left|\sigma^{\star}\right|^{\frac{1}{n^{\star}(x)}}+1 \quad \text { in } Q
$$

which using (2.33) and (4.28) is equivalent to

$$
c^{0}\left(x, \theta^{0}\right) \frac{\partial \theta^{0}}{\partial t}=\sigma^{0} \frac{\partial v^{0}}{\partial x} \quad \text { in } Q
$$

We passed to the limit in (2.3).

Let us complete this proof by giving another formula, equivalent to (4.29), for the definition of the homogenized heat coefficient $c^{0}$. Writing (4.29) at the point $s=\hat{Y}^{0}(x, r)$ yields

$$
c^{0}\left(x, \hat{Y}^{0}(x, r)\right)=\frac{\frac{\partial \hat{Z}^{0}}{\partial s}\left(x, \hat{Y}^{0}(x, r)\right)}{\left(\mu^{0}\left(x, \hat{Y}^{0}(x, r)\right)\right)^{\frac{1}{n^{\star}(x)}}} \text { in } \Omega \times \mathbf{R},
$$

in which we use the chain rule applied to the identity $\hat{Z}^{0}\left(x,\left(\hat{Y}^{0}(x, r)\right)=r\right.$, namely

$$
\left.\frac{\partial \hat{Z}^{0}}{\partial s}\left(x, \hat{Y}^{0}(x, r)\right) \frac{\partial \hat{Y}^{0}}{\partial r}(x, r)\right)=1 .
$$

This implies that $c^{0}$ defined by (4.29) can be equivalently defined by the formula

$$
c^{0}\left(x, \hat{Y}^{0}(x, r)\right)=\frac{1}{\frac{\partial \hat{Y}^{0}}{\partial r}(x, r)} \frac{1}{\left(\mu^{0}\left(x, \hat{Y}^{0}(x, r)\right)\right)^{\frac{1}{n^{\star}(x)}}} \quad \text { in } \Omega \times \mathbf{R} .
$$

In conclusion, we passed to the limit in (2.3) and (2.4), and therefore proved that in the setting of Proposition 4.1, the problem (2.1)-(2.8) is $\mathrm{SbH}$, 
with $n^{0}(x)=n^{\star}(x), c^{0}(x, \theta)$ defined by $(4.29)$ and $\psi^{0}(x, \gamma, \theta)=\mu^{0}(x, \theta)$, with $\mu^{0}(x, \theta)$ defined by (4.23). Note that the homogenized coefficients $c^{0}(x, \theta)$ and $\mu^{0}(x, \theta)$ satisfy $(2.10)-(2.16)$ (with constants possibly different of $M, \alpha$ and $\beta)$.

Remark 4.2 Definition of the homogenized heat and viscosity coefficients $c^{0}(x, \theta)$ and $\mu^{0}(x, \theta)$

Let us summarize in this Remark the way in which the homogenized coefficients $c^{0}(x, \theta)$ and $\mu^{0}(x, \theta)$ are defined in the thermoviscous case considered in Proposition 4.1.

From the data $c^{\varepsilon}(x, s), \mu^{\varepsilon}(x, s), n^{\star}(x)$ and $\theta_{0}(x)$, we define the function $\hat{Z}^{\varepsilon}(x, s)$ by

$$
\hat{Z}^{\varepsilon}(x, s)=\int_{\theta_{0}(x)}^{s} c^{\varepsilon}\left(x, s^{\prime}\right)\left(\mu^{\varepsilon}\left(x, s^{\prime}\right)\right)^{\frac{1}{n^{\star}(x)}} d s^{\prime} \quad \text { in } \Omega \times \mathbf{R}
$$

(see (4.7)). Then we extract a subsequence $\varepsilon^{\prime}$ such that the reciprocal functions $\left(\hat{Z}^{\varepsilon^{\prime}}\right)^{-1}(x, r)$ satisfy for every $r \in \mathbf{R}$ fixed

$$
\left(\hat{Z}^{\varepsilon^{\prime}}\right)^{-1}(x, r) \rightarrow\left(\hat{Z}^{0}\right)^{-1}(x, r) \quad \text { in } L^{\infty}(\Omega) \text { weak-star }
$$

for some function $\hat{Z}^{0}(x, s)$ (see (4.17) and (4.22)). From the same data, we also define (see (4.14), (4.18) and (4.24)) a function $\mu^{0}(x, s)$ such that for every $r \in \mathbf{R}$ fixed (and possibly for a further subsequence, still denoted by $\varepsilon^{\prime}$ ) one has

$\frac{1}{\left(\mu^{\varepsilon^{\prime}}\left(x,\left(\hat{Z}^{\varepsilon^{\prime}}\right)^{-1}(x, r)\right)\right)^{\frac{1}{n^{\star}(x)}}} \rightarrow \frac{1}{\left(\mu^{0}\left(x,\left(\hat{Z}^{0}\right)^{-1}(x, r)\right)\right)^{\frac{1}{n^{\star}(x)}}}$ in $L^{\infty}(\Omega)$ weak-star.

Finally from the functions $\hat{Z}^{0}(x, s)$ and $\mu^{0}(x, s)$, we define a function $c^{0}(x, s)$ by (4.29), i.e. by

$$
c^{0}(x, s)=\frac{\frac{\partial \hat{Z}^{0}}{\partial s}(x, s)}{\left(\mu^{0}(x, s)\right)^{\frac{1}{n^{\star}(x)}}} \quad \text { in } \Omega \times \mathbf{R}
$$


(an equivalent formula for $c^{0}(x, s)$ is (4.32)). The functions $\mu^{0}(x, s)$ and $c^{0}(x, s)$ depend only (but do depend) on the sequences $\mu^{\varepsilon}(x, s)$ and $c^{\varepsilon}(x, s)$, on the strain-rate sensitivity $n^{\star}(x)$ and on the initial condition $\theta^{0}(x)$. It does not depend on the other data $\left(f, \theta_{0}, \sigma_{a}\right.$ and $\left.v_{b}\right)$ of the problem.

Then $\mu^{0}(x, \theta)$ is the homogenized viscosity coefficient $\psi^{0}(x, \gamma, \theta)$ and $c^{0}(x, \theta)$ the homogenized heat coefficient for which problem $(2.1)-(2.8)$ is $\mathrm{SbH}$.

Remark 4.3 The case of a multiphase thermoviscous heterogeneous material made of periodic homogeneous layers

In this Remark we consider the special case of Proposition 4.1 where the heterogeneous thermoviscous material is made of periodic thin layers (of thickness of order $\varepsilon$ ) of homogeneous phases.

In other words, we consider here the case where (part of the notation in this Remark is the same as the notation in Remark 3.3)

$$
\begin{gathered}
n^{\star}(x)=n^{\star \star}, \\
c^{\varepsilon}(x, \theta)=\sum_{i} \chi_{i}\left(\frac{x}{\varepsilon}\right) c_{i}(\theta), \\
\mu^{\varepsilon}(x, \theta)=\sum_{i} \chi_{i}\left(\frac{x}{\varepsilon}\right) \mu_{i}(\theta),
\end{gathered}
$$

where $n^{\star \star}$ is given in $\mathbf{R}^{+}$, where the index $i$ runs between 1 and $I(I \geq 2$ denotes the number of phases), where $c_{i}: \mathbf{R} \rightarrow \mathbf{R}$ and $\mu_{i}: \mathbf{R} \rightarrow \mathbf{R}$ are heat and viscosity coefficients which do not depend on $x$ (and therefore describe homogeneous phases) and which satisfy respectively (2.11)-(2.12) and (2.15)-(2.16), where

$$
0=a_{0}<a_{1}<\ldots<a_{i-1}<a_{I}=1
$$


are given numbers and where $\chi_{i}$ is the characteristic function of the interval $\left(a_{i-1}, a_{i}\right)$ extended by periodicity to $\mathbf{R}$, i.e.

$$
\chi_{i}(x)= \begin{cases}1 & \text { if } k+a_{i-1}<x<k+a_{i} \quad \text { for some } k \in \mathbf{Z}, \\ 0 & \text { otherwise. }\end{cases}
$$

We set

$$
p_{i}=a_{i}-a_{i-1} .
$$

The number $p_{i}$ describes the volume fraction of the phase $i$ in the material and satisfies

$$
p_{i}>0 \quad \forall i, \quad \sum_{i} p_{i}=1
$$

Observe that

$$
\chi_{i}\left(\frac{x}{\varepsilon}\right) \rightarrow p_{i} \quad \text { in } L^{\infty}(\Omega) \text { weak-star. }
$$

We finally assume that

$$
\theta_{0}(x)=\theta^{\star \star} \text { in } \Omega
$$

where $\theta^{\star \star}$ is given in $\mathbf{R}$.

Hypotheses (2.9)-(2.22) are then satisfied, and the present setting is a particular case of Proposition 4.1. In this setting, the function $\hat{Z}^{\varepsilon}$ defined by (4.7) is given by

$$
\hat{Z}^{\varepsilon}(x, s)=\sum_{i} \chi_{i}\left(\frac{x}{\varepsilon}\right) \int_{\theta^{\star \star}}^{s} c_{i}\left(s^{\prime}\right)\left(\mu_{i}\left(s^{\prime}\right)\right)^{\frac{1}{n^{\star}}} d s^{\prime}=\sum_{i} \chi_{i}\left(\frac{x}{\varepsilon}\right) \hat{N}_{i}(s),
$$

where $\hat{N}_{i}: \mathbf{R} \rightarrow \mathbf{R}$ is the function defined by

$$
\hat{N}_{i}(s)=\int_{\theta^{\star \star}}^{s} c_{i}\left(s^{\prime}\right)\left(\mu_{i}\left(s^{\prime}\right)\right)^{\frac{1}{n^{\star \star}}} d s^{\prime} .
$$

Therefore the reciprocal function $\left(\hat{Z}^{\varepsilon}\right)^{-1}(x, r)$ is defined by

$$
\left(\hat{Z}^{\varepsilon}\right)^{-1}(x, r)=\sum_{i} \chi_{i}\left(\frac{x}{\varepsilon}\right)\left(\hat{N}_{i}\right)^{-1}(r),
$$


where $\left(\hat{N}_{i}\right)^{-1}: \mathbf{R} \rightarrow \mathbf{R}$ is the reciprocal function of the function $\hat{N}_{i}$.

In view of (4.39) the function $\left(\hat{Z}^{0}\right)^{-1}(x, r)=\hat{Y}^{0}(x, r)$ (which is defined by (4.17) and (4.22)) does not depend on $x$ and one has

$$
\left(\hat{Z}^{0}\right)^{-1}(r)=\hat{Y}^{0}(r)
$$

where

$$
\hat{Y}^{0}(r)=\sum_{i} p_{i}\left(\hat{N}_{i}\right)^{-1}(r)
$$

Similarly, the homogenized viscosity coefficient $\mu^{0}(x, s)$, which is defined by (4.14), (4.18) and (4.24), does not depend on $x$ : indeed, since the function $\zeta^{\varepsilon}(x, r)$ defined by $(4.14)$ is given here by

$$
\zeta^{\varepsilon}(x, r)=\frac{1}{\left(\mu^{\varepsilon}\left(x,\left(\hat{Z}^{\varepsilon}\right)^{-1}(x, r)\right)\right)^{\frac{1}{n^{\star}(x)}}}=\sum_{i} \frac{\chi_{i}\left(\frac{x}{\varepsilon}\right)}{\left(\mu_{i}\left(\left(\hat{N}_{i}\right)^{-1}(r)\right)\right)^{\frac{1}{n^{\star \star}}}}
$$

we deduce from (4.18), (4.22), (4.24), (4.39) and (4.46) that

$$
\frac{1}{\left(\mu^{0}\left(\hat{Y}^{0}(r)\right)\right)^{\frac{1}{n^{\star \star}}}}=\sum_{i} \frac{p_{i}}{\left(\mu_{i}\left(\left(\hat{N}_{i}\right)^{-1}(r)\right)\right)^{\frac{1}{n^{\star \star}}}} .
$$

Finally, we use formula (4.32) to compute the homogenized heat coefficient $c^{0}$. Since $\hat{Y}^{0}(x, r)$ and $\mu^{0}(x, s)$ do not depend on $x$, the homogenized heat coefficient is independent on $x$ and formula (4.32) combined with (4.45) and (4.47) yields

$$
c^{0}\left(\hat{Y}^{0}(r)\right)=\frac{\sum_{i} \frac{p_{i}}{\left(\mu_{i}\left(\left(\hat{N}_{i}\right)^{-1}(r)\right)\right)^{\frac{1}{n^{\star \star}}}}}{\sum_{i} p_{i} \frac{\partial\left(\hat{N}_{i}\right)^{-1}}{\partial r}(r)} .
$$

In the special setting of this Remark, which is concerned with periodic heterogeneous thermoviscous materials made of thin layers of homogeneous 
phases, equations (4.47) and (4.48) combined with (4.45) and (4.42) provide explicit formulas for the homogenized viscosity coefficient $\mu^{0}(x, \theta)$ and for the homogenized heat coefficient $c^{0}(x, \theta)$.

Remark 4.4 The case of power laws for a multiphase thermoviscous heterogeneous material made of periodic layers

Let us complete the previous Remark by an explicit example.

In the setting of Remark 4.3, consider the case where the thermoviscous material is made of periodic layers of $I$ phases with volume fractions $p_{i}$, which are characterized by heat coefficients $c_{i}(s)$ and by viscosity coefficients $\mu_{i}(s)$ which satisfy hypotheses (2.11)-(2.12) and (2.15)-(2.16) respectively, and which are given by power laws in some interval $A \leq s \leq B$ of $\mathbf{R}^{+}$, i.e. which satisfy

$$
\begin{gathered}
c_{i}(s)=K_{i} s^{\xi_{i}}, \quad \forall s \quad \text { with } \quad A \leq s \leq B, \\
\mu_{i}(s)=M_{i} s^{\lambda_{i}}, \quad \forall s \quad \text { with } \quad A \leq s \leq B,
\end{gathered}
$$

where $K_{i}$ and $M_{i}$ are given in $\mathbf{R}^{+}$, where $\xi_{i}$ and $\lambda_{i}$ are given in $\mathbf{R}$ and where $A$ and $B$ are given with $0<A<B<+\infty$. Note that heat and viscosity coefficients defined by power laws on the whole of $\mathbf{R}$ or even of $\mathbf{R}^{+}$would satisfy neither hypotheses (2.11)-(2.12) nor hypotheses (2.15)-(2.16); this is the reason why we assume that the heat and viscosity coefficients $c_{i}(s)$ and $\mu_{i}(s)$ of the phases are given by power laws only in the interval $A \leq s \leq B$. On the other hand, since we can have $A=\delta$ and $B=1 / \delta$ with $\delta>0$ small, the heat and viscosity coefficients can be defined as power laws on a very large part of $\mathbf{R}^{+}$.

We will assume that the powers $\xi_{i}, \lambda_{i}$ and the initial temperature $\theta^{\star \star}$ satisfy

$$
\left(1+\xi_{i}\right) n^{\star \star}+\lambda_{i} \neq 0
$$




$$
\theta^{\star \star} \leq A
$$

These two hypotheses are only technical. Note that in (4.49) and (4.50) the power laws could be replaced by exponential or logarithmic laws. Let us emphasize that the powers $\xi_{i}$ and $\lambda_{i}$ are assumed to be neither positive nor negative. This allows us to consider both softening and hardening processes, as well as problems with softening and hardening processes competing each other.

In this example the function $\hat{N}_{i}(s)$ defined by $(4.42)$ is given by

$$
\hat{N}_{i}(s)=\hat{g}_{i} s^{\frac{\left(1+\xi_{i}\right) n^{\star \star}+\lambda_{i}}{n^{\star \star}}}-\hat{K}_{i}, \quad \forall s \quad \text { with } \quad A \leq s \leq B
$$

(in the case where hypothesis (4.51) does not hold true, the power $\frac{\left(1+\xi_{i}\right) n^{\star \star}+\lambda_{i}}{n^{\star \star}}$ has to be replaced by a logarithm), where the constants $\hat{g}_{i}$ and $\hat{K}_{i}$ are given by

$$
\begin{gathered}
\hat{g}_{i}=\frac{n^{\star \star}}{\left(1+\xi_{i}\right) n^{\star \star}+\lambda_{i}} K_{i} M_{i}^{\frac{1}{n^{\star \star}}}, \\
\hat{K}_{i}=\hat{g}_{i} A^{\frac{\left(1+\xi_{i}\right) n^{\star \star}+\lambda_{i}}{n^{\star \star}}}-\int_{\theta^{\star \star}}^{A} c_{i}\left(s^{\prime}\right)\left(\mu_{i}\left(s^{\prime}\right)\right)^{\frac{1}{n^{\star \star}}} d s^{\prime} .
\end{gathered}
$$

Note that

$$
\hat{K}_{i}=\hat{g}_{i} A^{\frac{\left(1+\xi_{i}\right) n^{\star \star}+\lambda_{i}}{n^{\star \star}}} \quad \text { when } \quad A=\theta^{\star \star} .
$$

The reciprocal function $\left(\hat{N}_{i}\right)^{-1}(r)$ therefore satisfies

$$
\begin{gathered}
\left(\hat{N}_{i}\right)^{-1}(r)=\left(\frac{1}{\hat{g}_{i}}\left(r+\hat{K}_{i}\right)\right)^{\frac{n^{\star \star}}{\left(1+\xi_{i}\right) n^{\star \star}+\lambda_{i}}}, \\
\forall r \quad \text { with } \quad \hat{N}_{i}(A) \leq r \leq \hat{N}_{i}(B) .
\end{gathered}
$$

Since the function $\hat{N}_{i}(s)$ is strictly increasing and since $\hat{N}_{i}\left(\theta^{\star \star}\right)=0$, hypothesis (4.52) implies that $0<\hat{N}_{i}(A)<\hat{N}_{i}(B)<+\infty$. We will moreover assume that there exist $C$ and $D$ such that

$$
\min _{i} \hat{N}_{i}(A) \leq C<D \leq \max _{i} \hat{N}_{i}(B)
$$


Then on the interval $C \leq r \leq D$, the function $\hat{Y}^{0}(r)$ defined by (4.45) is given by

$$
\hat{Y}^{0}(r)=\sum_{i} p_{i}\left(\frac{1}{g_{i}}\left(r+\hat{K}_{i}\right)\right)^{\frac{n^{\star \star}}{\left(1+\xi_{i}\right) n^{\star \star}+\lambda_{i}}}, \quad \forall r \quad \text { with } \quad C \leq r \leq D
$$

and formula (4.47) which defines the homogenized viscosity coefficient $\mu^{0}$ reads as

$$
\begin{gathered}
\frac{1}{\left(\mu^{0}\left(\hat{Y}^{0}(r)\right)\right)^{\frac{1}{n^{\star \star}}}}=\sum_{i} \frac{p_{i}}{M_{i}^{\frac{1}{n^{\star \star}}}\left(\frac{1}{\hat{g}_{i}}\left(r+\hat{K}_{i}\right)\right)^{\frac{\lambda_{i}}{\left(1+\xi_{i}\right) n^{\star \star}+\lambda_{i}}}} \\
\forall r \quad \text { with } \quad C \leq r \leq D .
\end{gathered}
$$

Similarly, using (4.59) and the definition (4.56) of $\left(\hat{N}_{i}\right)^{-1}(r)$, which implies that

$$
\begin{gathered}
\frac{\partial\left(\hat{N}_{i}\right)^{-1}}{\partial r}(r)=\frac{n^{\star \star}}{\left(1+\xi_{i}\right) n^{\star \star}+\lambda_{i}} \frac{1}{\hat{g}_{i}}\left(\frac{1}{\hat{g}_{i}}\left(r+\hat{K}_{i}\right)\right)^{-\frac{\xi_{i} n^{\star \star}+\lambda_{i}}{\left(1+\xi_{i}\right) n^{\star \star}+\lambda_{i}}} \\
\forall r \quad \text { with } \hat{N}_{i}(A) \leq r \leq \hat{N}_{i}(B),
\end{gathered}
$$

formula (4.48) which defines the homogenized heat coefficient $c^{0}$ reads as

$$
\begin{gathered}
c^{0}\left(\hat{Y}^{0}(r)\right)=\frac{\sum_{i} \frac{p_{i}}{M_{i}^{\frac{1}{n^{\star \star}}}\left(\frac{1}{\hat{g}_{i}}\left(r+\hat{K}_{i}\right)\right)^{\frac{\lambda_{i}}{\left(1+\xi_{i}\right) n^{\star \star}+\lambda_{i}}}}}{\sum_{i} p_{i} \frac{n^{\star \star}}{\left(1+\xi_{i}\right) n^{\star \star}+\lambda_{i}} \frac{1}{\hat{g}_{i}}\left(\frac{1}{\hat{g}_{i}}\left(r+\hat{K}_{i}\right)\right)^{-\frac{\xi_{i} n^{\star \star}+\lambda_{i}}{\left(1+\xi_{i}\right) n^{\star \star}+\lambda_{i}}}} \\
\forall r \quad \text { with } \quad C \leq r \leq D .
\end{gathered}
$$

Formulas (4.59) and (4.60) combined with (4.58) explicitly give the values of the homogenized viscosity and heat coefficients $\mu^{0}(s)$ and $c^{0}(s)$ in the interval $\hat{Y}^{0}(C) \leq s \leq \hat{Y}^{0}(D)$. Observe that these homogenized coefficient 
are no more given by power laws in this interval, which covers a very large part of $\mathbf{R}^{+}$when $A=\delta$ and $B=1 / \delta$ with $\delta>0$ small and when $\theta^{\star \star}$ is close to zero. Therefore power laws are not $\mathrm{SbH}$.

\section{Problem (2.1)-(2.8) is $\mathrm{SbH}$ for a general viscosity coefficient $\psi^{\varepsilon}(x, \gamma, \theta)$ when $\sigma^{\star}(t, x)$ does not depend on $t$ (general thermovis- coplastic case with steady stress)}

Recall that by (2.23) one has

$$
\sigma^{\varepsilon}=\sigma^{\star} \quad \text { in } Q
$$

where $\sigma^{\star}$ is given by (2.27). In the special case where $\sigma^{\star}$ does not depend on the time, i.e. when

$$
\sigma^{\star}(t, x)=\sigma^{\star \star}(x),
$$

for some $\sigma^{\star \star}$, equation $(2.3)$ on $\theta^{\varepsilon}$ reads as

$$
c^{\varepsilon}\left(x, \theta^{\varepsilon}\right) \frac{\partial \theta^{\varepsilon}}{\partial t}=\sigma^{\star \star}(x) \frac{\partial \gamma^{\varepsilon}}{\partial t} \quad \text { in } Q .
$$

Note that $\sigma^{\star \star} \in L^{\infty}(\Omega)$ in view of (2.9), (2.21), (2.27) and (5.1), and that condition (5.1) is in particular satisfied when the boundary shearing $\sigma_{a}$ and the body force $g$ do not depend on time.

Defining the function $C^{\varepsilon}: \Omega \times \mathbf{R} \rightarrow \mathbf{R}$ by

$$
C^{\varepsilon}(x, s)=\int_{\theta_{0}(x)}^{s} c^{\varepsilon}\left(x, s^{\prime}\right) d s^{\prime} \quad \text { in } \Omega \times \mathbf{R},
$$

equation (5.2) is equivalent to

$$
C^{\varepsilon}\left(x, \theta^{\varepsilon}(t, x)\right)=\sigma^{\star \star}(x)\left(\gamma^{\varepsilon}(t, x)-\gamma_{0}(x)\right),
$$


when (5.1) holds true.

If we moreover assume that $\sigma^{\star \star}(x)$ is bounded away from zero, i.e. that

$$
\left|\sigma^{\star \star}(x)\right| \geq \hat{\alpha} \quad \text { a.e. } \quad x \in \Omega,
$$

for some $\hat{\alpha}>0,(5.4)$ is equivalent to

$$
\gamma^{\varepsilon}(t, x)=\frac{C^{\varepsilon}\left(x, \theta^{\varepsilon}(t, x)\right)}{\sigma^{\star \star}(x)}+\gamma_{0}(x),
$$

i.e. the strain $\gamma^{\varepsilon}$ is an explicit function of the temperature $\theta^{\varepsilon}$. Therefore, in the special case where (5.1) and (5.5) hold true, the general setting where the viscosity coefficient $\psi^{\varepsilon}(x, \gamma, \theta)$ depends on both $\gamma$ and $\theta$ can be reduced to the special case where the viscosity coefficient $\psi^{\varepsilon}$ depends only on $\theta$ by eliminating $\gamma$ in function of $\theta$ by the formula

$$
\gamma=\frac{C^{\varepsilon}(x, \theta)}{\sigma^{\star \star}(x)}+\gamma_{0}(x)
$$

(see (5.6)) and by defining a function $\mu^{\varepsilon}(x, \theta)$ by

$$
\mu^{\varepsilon}(x, \theta)=\psi^{\varepsilon}\left(x, \frac{C^{\varepsilon}(x, \theta)}{\sigma^{\star \star}(x)}+\gamma_{0}(x), \theta\right) .
$$

The following result is then an immediate consequence of Proposition 4.1.

Proposition 5.1 Assume that hypotheses (2.9)-(2.22) hold true and moreover that (see (2.27) for the definition of $\sigma^{\star}$ )

$$
\begin{gathered}
\sigma^{\star}(t, x)=\sigma^{\star \star}(x), \\
n^{\varepsilon}(x)=n^{\star}(x),
\end{gathered}
$$

for some $\sigma^{\star \star}(x) \in L^{\infty}(\Omega)$ which satisfies (5.5) and for some given $n^{\star}(x)$. Then the viscosity function $\psi^{\varepsilon}(x, \gamma, \theta)$ can be written as a viscosity function 
$\mu^{\varepsilon}(x, \theta)$ which is independent of $\gamma($ see $((5.8)))$, problem (2.1)-(2.8) is SbH and one has

$$
\begin{gathered}
n^{0}(x)=n^{\star}(x), \\
\psi^{0}(x, \gamma, \theta)=\mu^{0}(x, \theta),
\end{gathered}
$$

where the homogenized heat coefficient $c^{0}(x, \theta)$ and the homogenized viscosity coefficient $\mu^{0}(x, \theta)$ depend only on the sequences $c^{\varepsilon}(x, \theta)$ and $\psi^{\varepsilon}(x, \gamma, \theta)$, on the strain-rate sensitivity $n^{\star}(x)$, on the initial temperature and strain $\theta^{0}(x)$ and $\gamma^{0}(x)$ and on the function $\sigma^{\star}(x)$, which itself depends on the boundary shearing $\sigma_{a}(t)$ and on the body force $g(t, x)$. The homogenized coefficients $c^{0}(x, \theta)$ and $\mu^{0}(x, \theta)$ are given by the formulas summarized in Remark 4.2 for the viscosity coefficients $\mu^{\varepsilon}(x, \theta)$ defined by formula (5.8).

Remark 5.2 In the context of Proposition 5.1, the homogenized viscosity coefficient $\mu^{0}(x, \theta)$ can not in general be expressed as a function $\psi^{0}(x, \gamma, \theta)$ involving $\gamma$

The homogenized viscosity coefficient $\psi^{0}$ obtained in Proposition 5.1 is of the form $\mu^{0}(x, \theta)$, and therefore only depends on $\theta$. It is in general impossible to express this homogenized coefficient $\mu^{0}(x, \theta)$ as an equivalent function $\psi^{0}(x, \gamma, \theta)$ depending on both $\theta$ and $\gamma$. Indeed, it is in general impossible to pass to the limit in formula (5.4) (or in one of its equivalent possible forms) since $\theta^{\varepsilon}$ (as well as $\gamma^{\varepsilon}$ ) only weakly converges (at least as far as $x$ is concerned). Therefore in contrast with the case described in Remark 5.4 below, there is in general no explicit relation between $\theta^{0}$ and $\gamma^{0}$ available in the context of Proposition 5.1.

In formula (5.8), we eliminated $\gamma$ in function of $\theta$ in $\psi^{\varepsilon}(x, \gamma, \theta)$. But since the function $s \in \mathbf{R} \rightarrow C^{\varepsilon}(x, s) \in \mathbf{R}$ is one-to-one, one can equivalently 
rewrite equation (5.4) as

$$
\theta^{\varepsilon}(t, x)=\left(C^{\varepsilon}\right)^{-1}\left(x, \sigma^{\star \star}(x)\left(\gamma^{\varepsilon}(t, x)-\gamma_{0}(x)\right)\right) .
$$

One then defines from the viscosity coefficient $\psi^{\varepsilon}(x, \gamma, \theta)$ a viscosity coefficient $\nu^{\varepsilon}(x, \gamma)$ which does not depend on $\theta$ by eliminating $\theta$ in function of $\gamma$ by the formula

$$
\theta=\left(C^{\varepsilon}\right)^{-1}\left(x, \sigma^{\star \star}(x)\left(\gamma-\gamma_{0}(x)\right)\right),
$$

(see $(5.13))$ and by defining $\nu^{\varepsilon}(x, \gamma)$ by

$$
\nu^{\varepsilon}(x, \gamma)=\psi^{\varepsilon}\left(x, \gamma,\left(C^{\varepsilon}\right)^{-1}\left(x, \sigma^{\star \star}(x)\left(\gamma-\gamma_{0}(x)\right)\right)\right)
$$

It is not necessary to assume that (5.5) holds true to obtain (5.15). We nevertheless prefer to eliminate $\gamma$ in function of $\theta$, obtaining (5.8), and not $\theta$ in function of $\gamma$, obtaining (5.15), because the hypotheses of Proposition 3.1 impose to the heat coefficient to satisfy $c^{\varepsilon}(x, \theta)=c^{\star}(x)$ (hypothesis (3.2)), a restriction which has not to be made when the viscosity coefficient is of the form $\mu^{\varepsilon}(x, \theta)$.

When the restriction $c^{\varepsilon}(x, \theta)=c^{\star}(x)$ is enforced, then $C^{\varepsilon}(x, s)$ defined by (5.3) becomes

$$
C^{\varepsilon}(x, s)=c^{\star}(x)\left(s-\theta_{0}(x)\right),
$$

and equation (5.2) reads as

$$
c^{\star}(x)\left(\theta^{\varepsilon}(t, x)-\theta_{0}(x)\right)=\sigma^{\star \star}(x)\left(\gamma^{\varepsilon}(t, x)-\gamma_{0}(x)\right) .
$$

Then (5.14) reads as

$$
\theta=\frac{\sigma^{\star \star}(x)}{c^{\star}(x)}\left(\gamma-\gamma_{0}(x)\right)+\theta_{0}(x),
$$

and $\nu^{\varepsilon}(x, \gamma)$ defined by (5.15) becomes

$$
\nu^{\varepsilon}(x, \gamma)=\psi^{\varepsilon}\left(x, \gamma, \frac{\sigma^{\star \star}(x)}{c^{\star}(x)}\left(\gamma-\gamma_{0}(x)\right)+\theta_{0}(x)\right) .
$$


The fact that the restriction $c^{\varepsilon}(x, \theta)=c^{\star}(x)$ has to be made in the case where the temperature $\theta$ is eliminated in function of the strain $\gamma$ suggests that in mechanics it is not always suitable to consider the temperature as a hidden variable, because of the loss of information on the temperature dependent coefficients $c^{\varepsilon}(x, \theta)$ and $c^{0}(x, \theta)$.

The following result is then an immediate consequence of Proposition 3.1.

Proposition 5.3 Assume that hypotheses (2.9)-(2.22) hold true and moreover that (see (2.27) for the definition of $\sigma^{\star}$ )

$$
\begin{gathered}
\sigma^{\star}(t, x)=\sigma^{\star \star}(x), \\
n^{\varepsilon}(x)=n^{\star}(x), \\
c^{\varepsilon}(x, \theta)=c^{\star}(x),
\end{gathered}
$$

for some $\sigma^{\star \star}(x) \in L^{\infty}(\Omega)$ and for some given $n^{\star}(x), c^{\star}(x)$. Then the viscosity function $\psi^{\varepsilon}(x, \gamma, \theta)$ can be written as a viscosity function $\nu^{\varepsilon}(x, \gamma)$ which is independent of $\theta$ (see (5.18)), problem (2.1)-(2.8) is SbH and one has

$$
\begin{gathered}
n^{0}(x)=n^{\star}(x), \\
c^{0}(x, \theta)=c^{\star}(x), \\
\psi^{0}(x, \gamma, \theta)=\nu^{0}(x, \gamma),
\end{gathered}
$$

where the homogenized viscosity coefficient $\nu^{0}(x, \gamma)$ depends only on the sequence $\psi^{\varepsilon}(x, \gamma, \theta)$, on the heat coefficient $c^{\star}(x)$, on the strain-rate sensitivity $n^{\star}(x)$, on the initial temperature and strain $\theta^{0}(x)$ and $\gamma^{0}(x)$ and on the function $\sigma^{\star \star}(x)$, which itself depends on the boundary shearing $\sigma_{a}(t)$ and on the body force $g(t, x)$. The homogenized coefficient $\nu^{0}(x, \gamma)$ is given by the formula summarized in Remark 3.2 above for the viscosity coefficients $\nu^{\varepsilon}(x, \gamma)$ defined by formula (5.18). 
Remark 5.4 In the context of Proposition 5.3, the homogenized viscosity coefficient $\nu^{0}(x, \gamma)$ can equivalently be expressed as a function $\mu^{0}(x, \theta)$

In the special case of Proposition 5.3, relation (5.17) is affine in $\theta^{\varepsilon}$ and $\gamma^{\varepsilon}$ with coefficients which do not depend on $\varepsilon$. Therefore we can pass to the limit in (5.17). This gives

$$
c^{\star}(x)\left(\theta^{0}(t, x)-\theta_{0}(x)\right)=\sigma^{\star \star}(x)\left(\gamma^{0}(t, x)-\gamma_{0}(x)\right),
$$

which yields, when $\sigma^{\star \star}$ satisfies (5.5),

$$
\gamma^{0}(t, x)=\frac{c^{\star}(x)}{\sigma^{\star \star}(x)}\left(\theta^{0}(t, x)-\theta_{0}(x)\right)+\gamma_{0}(x) .
$$

Eliminating $\gamma$ in function of $\theta$ by the formula

$$
\gamma=\frac{c^{\star}(x)}{\sigma^{\star \star}(x)}\left(\theta-\theta_{0}(x)\right)+\gamma_{0}(x),
$$

one can then express the homogenized coefficient $\nu^{0}(x, \gamma)$ as a function $\mu^{0}(x, \theta)$ by setting

$$
\mu^{0}(x, \theta)=\nu^{0}\left(x, \frac{c^{\star}(x)}{\sigma^{\star \star}(x)}\left(\theta-\theta_{0}(x)\right)+\gamma_{0}(x)\right) .
$$

In the case where the formula given in Remark 3.2 for $\nu^{0}(x, \gamma)$ is explicit, one can also partially replace $\gamma$ by the affine function of $\theta$ defined by (5.26) in the explicit formula for $\nu^{0}(x, \gamma)$, obtaining an homogenized viscosity coefficient $\psi^{0}(x, \gamma, \theta)$. Nevertheless this process is in some sense artificial.

Remark 5.5 The case where $\sigma^{\star}$ depends on the time

In order to reduce the general problem to the special case where the viscosity coefficient $\psi^{\varepsilon}(x, \gamma, \theta)$ is of the form $\mu^{\varepsilon}(x, \theta)$ (or of the form $\nu^{\varepsilon}(x, \gamma)$ ), we have assumed that $\sigma^{\star}$ does not depend on $t$ (hypothesis (5.1)). If we do 
not make this hypothesis, but assume that $\sigma^{\star}$ now depends on $t$ and is smooth (say $\sigma^{\star} \in C^{1}(\bar{Q})$ ), equation (2.3), combined with (2.2) and (2.33), reads as

$$
c^{\varepsilon}\left(x, \theta^{\varepsilon}\right) \frac{\partial \theta^{\varepsilon}}{\partial t}=\sigma^{\star}(t, x) \frac{\partial \gamma^{\varepsilon}}{\partial t}=\frac{\partial\left(\sigma^{\star}(t, x) \gamma^{\varepsilon}\right)}{\partial t}-\frac{\partial \sigma^{\star}}{\partial t}(t, x) \gamma^{\varepsilon} \quad \text { in } Q
$$

which combined with the initial conditions (2.5) and (2.6) and with the definition (5.3) of $C^{\varepsilon}$ yields

$$
C^{\varepsilon}\left(x, \theta^{\varepsilon}(t, x)\right)=\sigma^{\star}(t, x) \gamma^{\varepsilon}(t, x)-\sigma^{\star}(0, x) \gamma_{0}(x)-\int_{0}^{t} \frac{\partial \sigma^{\star}}{\partial t}\left(t^{\prime}, x\right) \gamma^{\varepsilon}\left(t^{\prime}, x\right) d t^{\prime},
$$

which is equivalent to

$$
\begin{aligned}
\left.\theta^{\varepsilon}(t, x)\right)=\left(C^{\varepsilon}\right)^{-1}\left(x, \sigma^{\star}(t, x) \gamma^{\varepsilon}(\right. & t, x)-\sigma^{\star}(0, x) \gamma_{0}(x)- \\
& \left.-\int_{0}^{t} \frac{\partial \sigma^{\star}}{\partial t}\left(t^{\prime}, x\right) \gamma^{\varepsilon}\left(t^{\prime}, x\right) d t^{\prime}\right) .
\end{aligned}
$$

Formula (5.27) allows one to eliminate $\theta$ in function of $\gamma$ in the viscosity coefficient $\psi^{\varepsilon}(x, \gamma, \theta)$ by defining a viscosity coefficient $\varphi^{\varepsilon}$ by

$$
\begin{aligned}
& \varphi^{\varepsilon}(x,[\gamma](t, x))= \\
& =\psi^{\varepsilon}\left(x, \gamma(t, x),\left(C^{\varepsilon}\right)^{-1}\left(x, \sigma^{\star}(t, x) \gamma(t, x)-\sigma^{\star}(0, x) \gamma_{0}(x)-\right.\right. \\
& \left.\left.\quad-\int_{0}^{t} \frac{\partial \sigma^{\star}}{\partial t}\left(t^{\prime}, x\right) \gamma\left(t^{\prime}, x\right) d t^{\prime}\right)\right) .
\end{aligned}
$$

Observe however that formula (5.28) does not define the function $\varphi^{\varepsilon}$ from the pointwise value $\gamma(t, x)$ of $\gamma$ at the point $(t, x)$ but from all the values of $\gamma\left(t^{\prime}, x\right)$ for $t^{\prime}$ in the interval $0<t^{\prime}<t$, or more exactly from the values $\gamma(t, x)$ and $\int_{0}^{t} \frac{\partial \sigma^{\star}}{\partial t}\left(t^{\prime}, x\right) \gamma\left(t^{\prime}, x\right) d t^{\prime}$; this is the reason why we wrote $\varphi^{\varepsilon}(x,[\gamma](t, x))$ and not $\varphi^{\varepsilon}(x, \gamma(t, x))$ in the definition (5.28).

There is therefore a striking difference between the case where $\sigma^{\star}$ does not depend on $t$ (in this case one can write $\psi^{\varepsilon}(x, \gamma, \theta)$ as a coefficient $\mu^{\varepsilon}(x, \theta)$ 
defined by (5.8) or a coefficient $\nu^{\varepsilon}(x, \gamma)$ defined by (5.15)), and the case where $\sigma^{\star}$ depends on $t$ (in this case, a memory effect appears in the coefficient $\eta^{\varepsilon}(x,[\gamma](t, x))$ defined by $\left.(5.28)\right)$.

Remark 5.6 The case of a multiphase general thermoviscoplastic heterogeneous material made of periodic homogeneous layers when $\sigma^{\star}$ is steady

In this Remark we consider the special case of Proposition 5.1 where the heterogeneous thermoviscoplastic material is made of periodic thin layers (of thickness of order $\varepsilon$ ) of homogeneous phases.

In other words, we consider here the case where (part of the notation in this Remark is the same as the notation in Remarks 3.3 and 4.3)

$$
\begin{gathered}
n^{\star}(x)=n^{\star \star}, \\
c^{\varepsilon}(x, \theta)=\sum_{i} \chi_{i}\left(\frac{x}{\varepsilon}\right) c_{i}(\theta), \\
\psi^{\varepsilon}(x, \gamma, \theta)=\sum_{i} \chi_{i}\left(\frac{x}{\varepsilon}\right) \psi_{i}(\gamma, \theta),
\end{gathered}
$$

where $n^{\star \star}$ is given in $\mathbf{R}^{+}$, where the index $i$ runs between 1 and $I(I \geq 2$ denotes the number of phases), where $c_{i}: \mathbf{R} \rightarrow \mathbf{R}$ and $\psi_{i}: \mathbf{R} \rightarrow \mathbf{R}$ are heat and viscosity coefficients which do not depend on $x$ (and therefore describe homogeneous phases) and which satisfy respectively (2.11)-(2.12) and (2.14)-(2.16), where

$$
0=a_{0}<a_{1}<\ldots<a_{i-1}<a_{I}=1
$$

are given numbers and where $\chi_{i}$ is the characteristic function of the interval $\left(a_{i-1}, a_{i}\right)$ extended by periodicity to $\mathbf{R}$, i.e.

$$
\chi_{i}(x)= \begin{cases}1 & \text { if } \quad k+a_{i-1}<x<k+a_{i} \quad \text { for some } k \in \mathbf{Z} \\ 0 & \text { otherwise }\end{cases}
$$


We set

$$
p_{i}=a_{i}-a_{i-1}
$$

The number $p_{i}$ describes the volume fraction of the phase $i$ in the material and satisfies

$$
p_{i}>0 \quad \forall i, \quad \sum_{i} p_{i}=1 .
$$

Observe that

$$
\chi_{i}\left(\frac{x}{\varepsilon}\right) \rightarrow p_{i} \quad \text { in } L^{\infty}(\Omega) \text { weak-star. }
$$

We finally assume that

$$
\begin{aligned}
& \gamma_{0}(x)=\gamma^{\star \star} \quad \text { in } \Omega, \\
& \theta_{0}(x)=\theta^{\star \star} \quad \text { in } \Omega,
\end{aligned}
$$

where $\gamma^{\star \star}$ and $\theta^{\star \star}$ are given in $\mathbf{R}$.

In this setting the function $C^{\varepsilon}$ defined by (5.3) is given by

$$
C^{\varepsilon}(x, s)=\sum_{i} \chi_{i}\left(\frac{x}{\varepsilon}\right) C_{i}(s),
$$

where $C_{i}: \mathbf{R} \rightarrow \mathbf{R}$ is the function defined by

$$
C_{i}(s)=\int_{\theta^{\star \star}}^{s} c_{i}\left(s^{\prime}\right) d s^{\prime},
$$

and the viscosity coefficient $\mu^{\varepsilon}(x, s)$ defined by (5.8) is given by

$$
\mu^{\varepsilon}(x, s)=\sum_{i} \chi_{i}\left(\frac{x}{\varepsilon}\right) \mu_{i}(x, s)
$$

where $\mu_{i}: \Omega \times \mathbf{R} \rightarrow \mathbf{R}$ is the function defined by

$$
\mu_{i}(x, s)=\psi_{i}\left(\frac{C_{i}(s)}{\sigma^{\star \star}(x)}+\gamma^{\star \star}, s\right) .
$$


Hypotheses (2.9)-(2.22) are then satisfied, and the present setting is a particular case of Proposition 4.1. In this setting, the function $\hat{Z}^{\varepsilon}$ defined by (4.7) is given by

$$
\hat{Z}^{\varepsilon}(x, s)=\sum_{i} \chi_{i}\left(\frac{x}{\varepsilon}\right) \check{N}_{i}(x, s),
$$

where $\check{N}_{i}: \Omega \times \mathbf{R} \rightarrow \mathbf{R}$ is the function defined by

$$
\check{N}_{i}(x, s)=\int_{\theta^{\star \star}}^{s} c_{i}\left(s^{\prime}\right)\left(\mu_{i}\left(x, s^{\prime}\right)\right)^{\frac{1}{n^{\star \star}}} d s^{\prime} .
$$

Therefore the reciprocal function $\left(\hat{Z}^{\varepsilon}\right)^{-1}(x, r)$ is defined by

$$
\left(\hat{Z}^{\varepsilon}\right)^{-1}(x, r)=\sum_{i} \chi_{i}\left(\frac{x}{\varepsilon}\right)\left(\check{N}_{i}\right)^{-1}(x, r),
$$

where the function $r \in \mathbf{R} \rightarrow\left(\check{N}_{i}\right)^{-1}(x, r) \in \mathbf{R}$ is the reciprocal function of the function $s \in \mathbf{R} \rightarrow \check{N}_{i}(x, s) \in \mathbf{R}$ for $x$ fixed.

In view of (5.35) the function $\left(\hat{Z}^{0}\right)^{-1}(x, r)=\hat{Y}^{0}(x, r)$ (which is defined by (4.17) and (4.22)) is given by

$$
\hat{Y}^{0}(x, r)=\sum_{i} p_{i}\left(\check{N}_{i}\right)^{-1}(x, r) .
$$

Similarly, the function $\zeta^{\varepsilon}(x, r)$ defined by (4.14) is given here by

$$
\zeta^{\varepsilon}(x, r)=\sum_{i} \frac{\chi_{i}\left(\frac{x}{\varepsilon}\right)}{\left(\mu_{i}\left(\left(\check{N}_{i}\right)^{-1}(x, r)\right)\right)^{\frac{1}{n^{\star \star}}}} .
$$

From (4.18), (4.22), (4.24), (5.35) and (5.46) we deduce that

$$
\frac{1}{\left(\mu^{0}\left(\hat{Y}^{0}(x, r)\right)\right)^{\frac{1}{n^{\star}}}}=\sum_{i} \frac{p_{i}}{\left(\mu_{i}\left(\left(\check{N}_{i}\right)^{-1}(x, r)\right)\right)^{\frac{1}{n^{\star \star}}}} .
$$

Finally, we use formula (4.32) to compute the homogenized heat coefficient $c^{0}(x, s)$, which, combined with (5.45) and (5.46) yields

$$
c^{0}\left(\hat{Y}^{0}(x, r)\right)=\frac{\sum_{i} \frac{p_{i}}{\left(\mu_{i}\left(\left(\check{N}_{i}\right)^{-1}(x, r)\right)^{\frac{1}{n^{\star \star}}}\right.}}{\sum_{i} p_{i} \frac{\partial\left(\check{N}_{i}\right)^{-1}}{\partial r}(x, r)} .
$$


In the special setting of this Remark, which is concerned with periodic heterogeneous thermoviscoplastic materials made of thin layers of homogeneous phases when $\sigma^{\star}$ does not depend on $t$, equations (5.47) and (5.48) combined with (5.39), (5.41), (5.43) and (5.45) provide explicit formulas for the homogenized viscosity coefficient $\mu^{0}(x, \theta)$ and for the homogenized heat coefficient $c^{0}(x, \theta)$. Remark that, in general, the homogenized viscosity coefficient $\mu^{0}(x, \theta)$ cannot be expressed by an equivalent function $\psi^{0}(x, \gamma, \theta)$ depending on both $\gamma$ and $\theta$ (see Remark 5.2).

Remark 5.7 The case of power laws for a multiphase thermoviscoplastic heterogeneous material made of periodic layers: a numerical example

Let us complete the previous Remark by an explicit example.

In the setting of Remark 5.6, consider the case where the thermoviscopastic material is made of periodic layers of $I$ phases with volume fractions $p_{i}$, which are characterized by heat coefficients $c_{i}(\theta)$ and by viscosity coefficients $\psi_{i}(\gamma, \theta)$ which satisfy hypotheses $(2.11)-(2.12)$ and (2.14)-(2.16) respectively, and which are given by power laws in the intervals $A^{\prime} \leq \gamma \leq B^{\prime}$ and $A \leq \theta \leq B$, i.e. which satisfy

$$
\begin{gathered}
c_{i}(\theta)=K_{i} \theta^{\xi_{i}}, \quad \forall \theta \quad \text { with } \quad A \leq \theta \leq B, \\
\psi_{i}(\gamma, \theta)=G_{i} \gamma^{m_{i}} \theta^{\lambda_{i}}, \quad \forall(\gamma, \theta) \quad \text { with } \quad A^{\prime} \leq \gamma \leq B^{\prime}, \quad A \leq \theta \leq B,
\end{gathered}
$$

where $K_{i}$ and $G_{i}$ are given in $\mathbf{R}^{+}$, where $\xi_{i}, m_{i}$ and $\lambda_{i}$ are given in $\mathbf{R}$ and where $A, B, A^{\prime}$ and $B^{\prime}$ are given with $0<A<B<+\infty, 0<A^{\prime}<$ $<B^{\prime}<+\infty$. Note that heat and viscosity coefficients defined by power laws on the whole of $\mathbf{R}$ or even of $\mathbf{R}^{+}$would satisfy neither hypotheses (2.11)-(2.12) nor hypotheses (2.14)-(2.16); this is the reason why we assume that the heat and viscosity coefficients $c_{i}(\theta)$ and $\psi_{i}(\gamma, \theta)$ of the phases are 
given by power laws only in the intervals $A^{\prime} \leq \gamma \leq B^{\prime}, A \leq \theta \leq B$. On the other hand, since we can have $A=\delta, B=1 / \delta, A^{\prime}=\delta^{\prime}, B^{\prime}=1 / \delta^{\prime}$ with $\delta>0$ small and $\delta^{\prime}>0$ small, the heat and viscosity coefficients of the phases can be defined as power laws on a very large part of $\mathbf{R}^{+}$and $\left(\mathbf{R}^{+}\right)^{2}$. Note that in (5.49) and (5.50) the power laws could be replaced by exponential or logarithmic laws.

In this case the constitutive law (2.4) reads as

$$
\sigma=\sum_{i} \chi_{i}\left(\frac{x}{\varepsilon}\right) G_{i} \gamma^{m_{i}} \theta^{\lambda_{i}}\left|\frac{\partial v}{\partial x}\right|^{n^{\star \star}-1} \frac{\partial v}{\partial x}
$$

when $A^{\prime} \leq \gamma \leq B^{\prime}$ and $A \leq \theta \leq B$

We will assume that the steady stress $\sigma^{\star \star}$, the powers $\xi_{i}$, the initial strain and temperature $\gamma^{\star \star}$ and $\theta^{\star \star}$ and the numbers $A, B, A^{\prime}$ and $B^{\prime}$ satisfy

$$
\begin{gathered}
\sigma^{\star \star}(x)=\sigma^{\star \star}, \quad \sigma^{\star \star} \in \mathbf{R}^{+} \\
\left(1+\xi_{i}\right)>0, \quad \gamma^{\star \star}>0, \quad \theta^{\star \star}>0, \\
A=\theta^{\star \star}, \\
A^{\prime} \leq \frac{K_{i}}{\sigma^{\star \star}\left(1+\xi_{i}\right)}\left(A^{1+\xi_{i}}-\left(\theta^{\star \star}\right)^{1+\xi_{i}}\right)+\gamma^{\star \star}, \quad \forall i, \\
B^{\prime} \geq \frac{K_{i}}{\sigma^{\star \star}\left(1+\xi_{i}\right)}\left(B^{1+\xi_{i}}-\left(\theta^{\star \star}\right)^{1+\xi_{i}}\right)+\gamma^{\star \star}, \quad \forall i .
\end{gathered}
$$

In this example, under the above hypotheses, the function $C_{i}(s)$ defined by (5.39) is given by

$$
C_{i}(s)=\frac{K_{i}}{1+\xi_{i}}\left(s^{1+\xi_{i}}-\left(\theta^{\star \star}\right)^{1+\xi_{i}}\right)
$$

the function $\mu_{i}(x, s)$ defined by $(5.41)$ is given by

$$
\mu_{i}(x, s)=G_{i}\left(\frac{K_{i}}{\sigma^{\star \star}\left(1+\xi_{i}\right)}\left(s^{1+\xi_{i}}-\left(\theta^{\star \star}\right)^{1+\xi_{i}}\right)+\gamma^{\star \star}\right)^{m_{i}} s^{\lambda_{i}},
$$


while the function $\check{N}_{i}(x, s)$ defined by $(5.43)$ is given by $\check{N}_{i}(x, s)=K_{i} G_{i}^{\frac{1}{n^{\star \star}}} \int_{\theta^{\star \star}}^{s}\left(s^{\prime}\right)^{\frac{\xi_{i} n^{\star \star}+\lambda_{i}}{n^{\star \star}}}\left(\frac{K_{i}}{\sigma^{\star \star}\left(1+\xi_{i}\right)}\left(\left(s^{\prime}\right)^{1+\xi_{i}}-\left(\theta^{\star \star}\right)^{1+\xi_{i}}\right)+\gamma^{\star \star}\right)^{\frac{m_{i}}{n^{\star \star}}} d s^{\prime}$, for every $s$ with $A \leq s \leq B$. In general the reciprocal function $(\check{N})^{-1}(x, r)$ can not be computed explicitly, but we will obtain it numerically. Such will be also the case of the homogenized heat and viscosity coefficients $c^{0}(x, s)$ and $\mu^{0}(x, s)$.

More specifically, we consider a thermoviscoplastic material made of two metallic phases having densities

$$
\rho_{1}=7800 \mathrm{Kg} / \mathrm{m}^{3}, \quad \rho_{2}=7900 \mathrm{Kg} / \mathrm{m}^{3}
$$

moduli

$$
G_{1}=430 \mathrm{MPa}, \quad G_{2}=450 \mathrm{MPa},
$$

specific heat coefficients

$$
\eta_{1}=500 \quad \mathrm{~J} / \mathrm{Kg}^{0} \mathrm{~K}, \quad \eta_{2}=550 \quad \mathrm{~J} / \mathrm{Kg}^{0} \mathrm{~K}
$$

fraction of plastic work converted into heating

$$
\beta_{1}=0.9, \quad \beta_{2}=0.9
$$

Therefore $\xi_{i}=\xi_{2}=0$ and the heat coefficients take the values

$$
K_{1}=4.333333 \mathrm{MN} / \mathrm{m}^{2} \mathrm{~K}, \quad K_{2}=4.827778 \mathrm{MN} / \mathrm{m}^{2} \mathrm{~K} .
$$

The phases are assumed to exhibit thermal softening with powers

$$
\lambda_{1}=-0.48, \quad \lambda_{2}=-0.51
$$

strain hardening with powers

$$
m_{1}=0.09, \quad m_{2}=0.12
$$


and common strain-rate sensitivity

$$
n^{\star \star}=0.016 .
$$

Figures 3-8 present numerical results due to George Chatzigeorgiou, whose collaboration is gratefully acknowledged.

Figures 3 and 4 present the values of the functions $\left(\check{N}_{1}\right)^{-1}$ and $\left(\check{N}_{2}\right)^{-1}$ of the two phases for the values $200 \mathrm{MPa}, 400 \mathrm{MPa}$ and $800 \mathrm{MPa}$ of the stress $\sigma^{\star \star}$. The two figures only differ by the range considered for $r$. In Figure 3, $r$ takes its values between 0 and $3 \mathrm{e}+088=3.10^{88}$, while in Figure $4, r$ takes its values between 0 and $1 \mathrm{e}+084=10^{84}$ (the values of the variable in the horizontal axis have to be read according to the rule $k e+\ell=k 10^{\ell}$ ). For large values of $r$, the three curves of $\left(\check{N}_{1}\right)^{-1}$ and $\left(\check{N}_{2}\right)^{-1}$ seem to coincide independently of the values of $\sigma^{\star \star}$ (see Figure 3). In contrast, in Figure 4, namely at the onset of the deformation, the influence of the value of $\sigma^{\star \star}$ is clear at every stage of the deformation process and is much more important for the material with the larger thermal softening, which exhibits earlier shear banding ("blow-up" of temperature).

Figures 5 and 6 present the values of the viscosity coefficients $\mu_{1}$ and $\mu_{2}$ of the two phases and of the homogenized viscosity coefficient $\mu^{0}$ for the same values $200 \mathrm{MPa}, 400 \mathrm{MPa}$ and $800 \mathrm{MPa}$ of $\sigma^{\star \star}$. Here again, the three curves of $\mu_{1}, \mu_{2}$ and $\mu^{0}$ seem to coincide independently of the values of $\sigma^{\star \star}$ for $r$ large (see Figure 5), but they clearly differ at the onset of the deformation (see Figure 6). The weaker material exhibits a clear softening. We see again the same "attraction" by the worst material, in a ratio $3 / 1$.

Finally Figures 7 and 8 present the constant heat coefficients $c_{1}$ and $c_{2}$ of the two phases and the values of the homogenized heat coefficients for the same values $200 \mathrm{MPa}, 400 \mathrm{MPa}$ and $800 \mathrm{MPa}$ of $\sigma^{\star \star}$. Here again the three 
curves of $c^{0}$ seem to coincide indenpendtly of the values of $\sigma^{\star \star}$ for $r$ large (see Figure 7), but they clearly differ at the onset of the deformation (see Figure 8). The aforementioned "attraction" by the material with the larger thermal softening (weaker material) is now much more important, in a ratio $4.5 / 1$.

In conclusion, the above heterogeneous bimetallic material made of two homogeneous phases with volume fractions $\frac{1}{2}$ gives an homogenized behavior which is just a little better than the weaker of the two phases, almost like a chain whose strength is equal to the strength of the weaker link. The contribution of the stronger material seems to be less important, probably because of the fact that, in the one dimensional setting considered in this paper, one can not "surround" the weak material by the strong one, in contrast with what can be done in a two or three dimensional setting.

Acknowledgements. The numerical examples of this paper are due to George Chatzigeorgiou, whose collaboration is gratefully acknowledged. The results of the present work have been obtained during visits made by the first author to the Laboratoire Jacques-Louis Lions of the Université Pierre et Marie Curie (Paris VI) and by the second author to the Department of Civil Engineering of the Aristotle University of Thessaloniki. The support and hospitality of both institutions are gratefully acknowledged. The support of the Research Committee of the Aristotle University of Thessaloniki is also gratefully acknowledged. Finally the interest and support of UMT are sincerely acknowledged. 


\section{References}

Aboudi, J., Pindera, M., Arnold, S., 1999. Higher-order theory for functionally graded materials. Composites: Part B 30, 777-832.

Alshirts, V., Maugin, G., 2005. Dynamics of multilayers: elastic waves in an anisotropic graded or stratified plate. Wave Motion 41, 357-394.

Babuska, I., 1976a. Homogenization and its application. Mathematical and computational problems, in Numerical solution of partial differential equations-III, (SYNSPADE 1975), pp. 89-116. Academic Press.

Babuska, I., 1976b. Homogenization approach in engineering, in Computing methods in applied sciences and engineering (Second International Symposium, Versailles, 1975), Part 1, Lecture Notes in Economics and Mathematical Systems 134, pp. 137-153. Springer Verlag.

Bansal, Y., Pindera, M., 2003. Efficient reformulation of the thermoelastic higher-order theory for functionally graded materials. Journal of Thermal Stresses 26, 1055-1092.

Bansal, Y., Pindera, M., 2005. A second look at the higher-order theory for periodic multiphase materials. Journal of Applied Mechanics 72, 177-195.

Bardzokas, D., Zobnin, A., 2005. Mathematical modelling of physical processes in composite materials of periodical structures. URSS Editorial.

Batra, R., Love, B., 2006a. Determination of effective thermomechanical parameters of a mixture of two elastothermoviscoplastic constituents. International Journal of Plasticity 22, 1026-1061. 
Batra, R., Love, B., 2006b. Consideration of microstructural effects in the analysis of adiabatic shear bands in a tungsten heavy alloy. International Journal of Plasticity 22, 1858-1878.

Bensoussan, A., Lions, J.-L., Papanicolaou, G., 1978. Asymptotic methods for periodic structures. North Holland.

Charalambakis, N., Murat, F., 1989. Weak solutions to initial-boundary value problems for the shearing of non-homogeneous thermoviscoplastic materials. Proceedings of the Royal Society of Edinburgh 113A, 257-265.

Charalambakis, N., Murat, F., 2006a. Approximation by finite elements, existence and uniqueness for a model of stratified thermoviscoplastic materials. Ricerche di Matematica 55, 171-218.

Charalambakis, N., Murat, F., 2006b. Homogenization of stratified thermoviscoplastic materials. Quarterly of Applied Mathematics 64, 359-399.

Francfort, G., Leguillon, D., Suquet, P., 1983. Homogenization for linearly viscoelastic bodies. Comptes-Rendus de l'Académie des Sciences de Paris I 296, 287-290.

Francfort, G., Nguyen, Q., Suquet, P., 1983. Thermodynamics and the homogenized thermomechanical behavior. Comptes-Rendus de l'Académie des Sciences de Paris II 296, 1007-1010.

Geers, M., 1997. Experimental analysis and computational modelling of damage and fracture. Ph.D. thesis, Eindoven University of Technology.

Ghosh, S., Lee, K., Moorthy, S., 1995. Multiple scale analysis of heterogeneous elastic structures using homogenization theory and Voronoi cell 
finite element method. International Journal of Solids and Structures 32, $27-62$.

Ghosh, S., Lee, K., Raghavan, P., 2001. A multi-level computational model for multi-scale damage analysis in composite and porous materials. International Journal of Solids and Structures 38, 2335-2385.

Guinovart-Diaz, R., Rodriguez-Ramos, R., Bravo-Castillero, J., Maugin, G., 2005. A recursive asymptotic homogenization scheme for multi-phase fiberreinforced composites. Mechanics of Materials 37, 1119-1131.

Hashin, Z., 1983. Analysis of composite materials: A survey. Journal of Applied Mechanics 50, 481-505.

Idiart, M., Moulinec, H., Ponte-Castaneda, P., Suquet, P., 2006. Macroscopic behavior and field fluctuations in viscoplastic composites: Second-order estimates versus full-field simulations. Journal of the Mechanics and Physics of Solids 54, 1029-1063.

Lemaitre, J., Chaboche, J.-L., 2001. Mécanique des matériaux solides. Dunod.

Maugin, G., 1992. The thermomechanics of plasticity and fracture. Cambridge University Press.

Meuwissen, M., 1998. An inverse method for the mechanical characterization of metals. Ph.D. thesis, Eindhoven University of Technology.

Michel, J., Suquet, P., 2004. Computational analysis of nonlinear composites structures using the nonuniform transformation field analysis. Computer Methods in Applied Mechanics and Engineering 193, 5477-5502. 
Murat, F., 1977. H-convergence. Séminaire d'analyse fonctionnelle et numérique de l'Université d'Alger. Multicopied, 34 pages. English translation: Murat, F., Tartar, L., 1997, H-convergence, in Topics in the mathematical modelling of composite materials, ed. by A. Cherkaev and R.V. Kohn, Progress in Nonlinear Differential Equations and their Applications 31, pp. 21-43. Birkhäuser.

Nemat-Nasser, S., 1999. Averaging theorems in finite deformation plasticity. Mechanics of Materials 31, 493-523.

Sanchez-Palencia, E., 1978. Non-homogeneous media and vibration theory. Lecture Notes in Physics 127. Springer Verlag.

Shehadeh, M., Zbib, H., de la Rubia, T., 2005. Multiscale dislocation dynamics simulations. International Journal of Plasticity 21, 2369-2390.

Suquet, P., 1982. Plasticité et homogénéisation. Thèse d'Etat, Université Pierre et Marie Curie (Paris VI).

Suquet, P., 1983. Limit analysis and homogenization. Comptes-Rendus de l'Académie des Sciences de Paris II 296, 1335-1358.

Suquet, P., 2005. On the effect of small fluctuations in the volume fraction of constituents on the effective properties of composites. Comptes-Rendus de l'Académie des Sciences de Paris, Mécanique 333, 219-266.

Tartar, L., 1977. Homogénéisation et compacité par compensation. Cours Peccot, Collège de France. Partially written in Murat, F. (1977).

Van der Sluis, O., Schreurs, P., Meijer, H., 1999. Effective properties of a viscoplastic constitutive model obtained by homogenisation. Mechanics of Materials 31, 743-759. 
Wright, T., 2002. The physics and mathematics of shear bands. Cambridge University Press. 


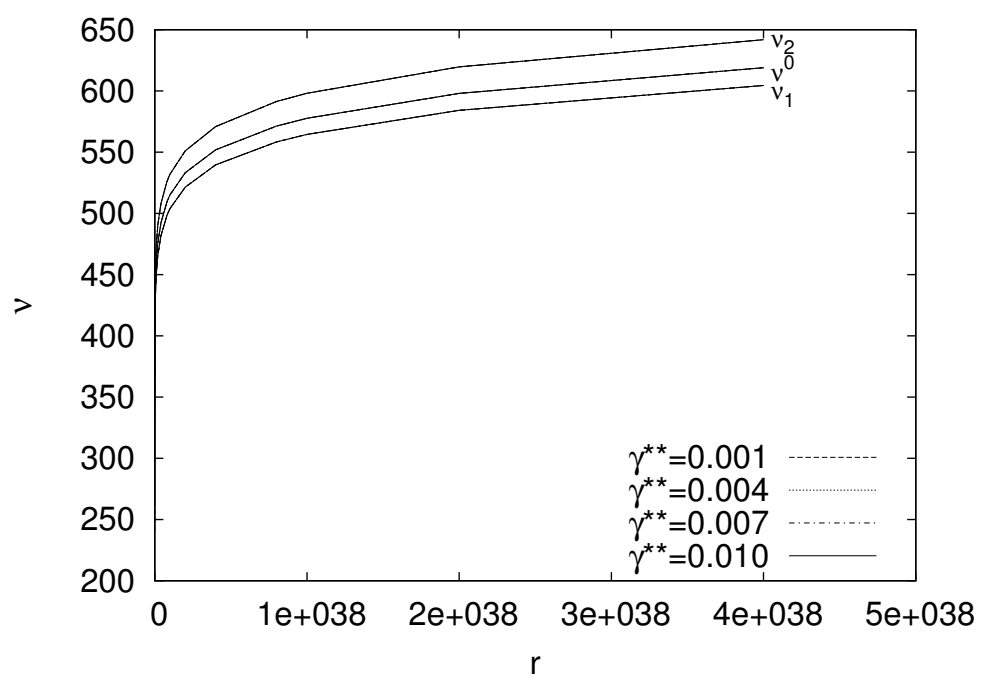

Figure 1: The hardenings $\nu_{1}$ and $\nu_{2}$ of the phases and the homogenized hardening $\nu^{0}$ for different values of the initial strain $\gamma^{\star \star}$ (the curves seem to coincide for the four values of $\gamma^{\star \star}$ )

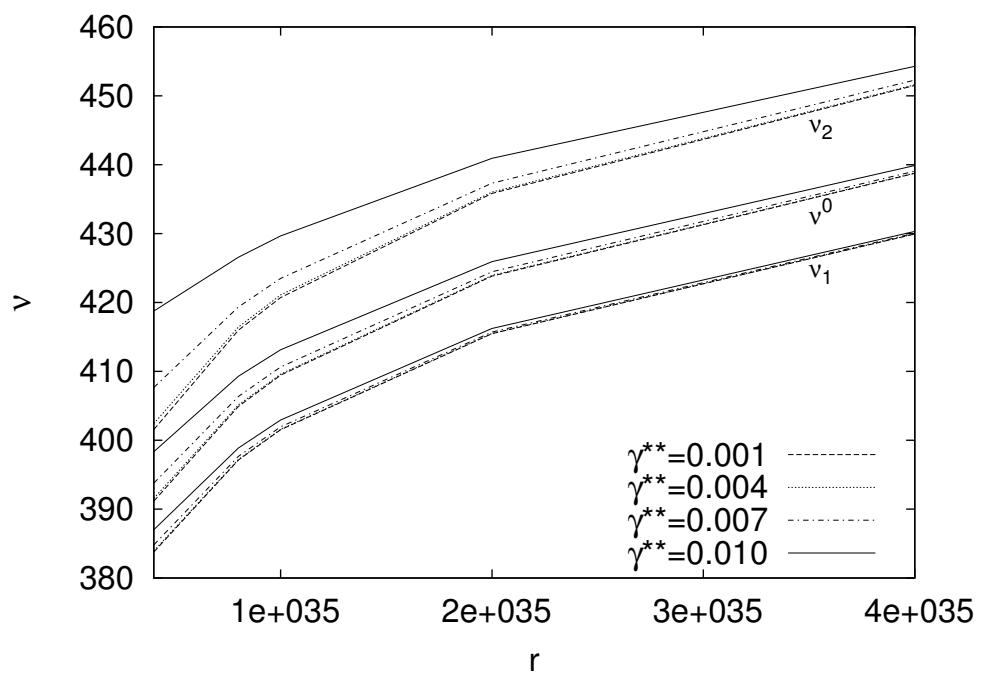

Figure 2: The hardenings $\nu_{1}$ and $\nu_{2}$ of the phases and the homogenized hardening $\nu^{0}$ for different values of the initial strain $\gamma^{\star \star}$ at the onset of the deformation (the curves differ for the four values of $\gamma^{\star \star}$ ) 


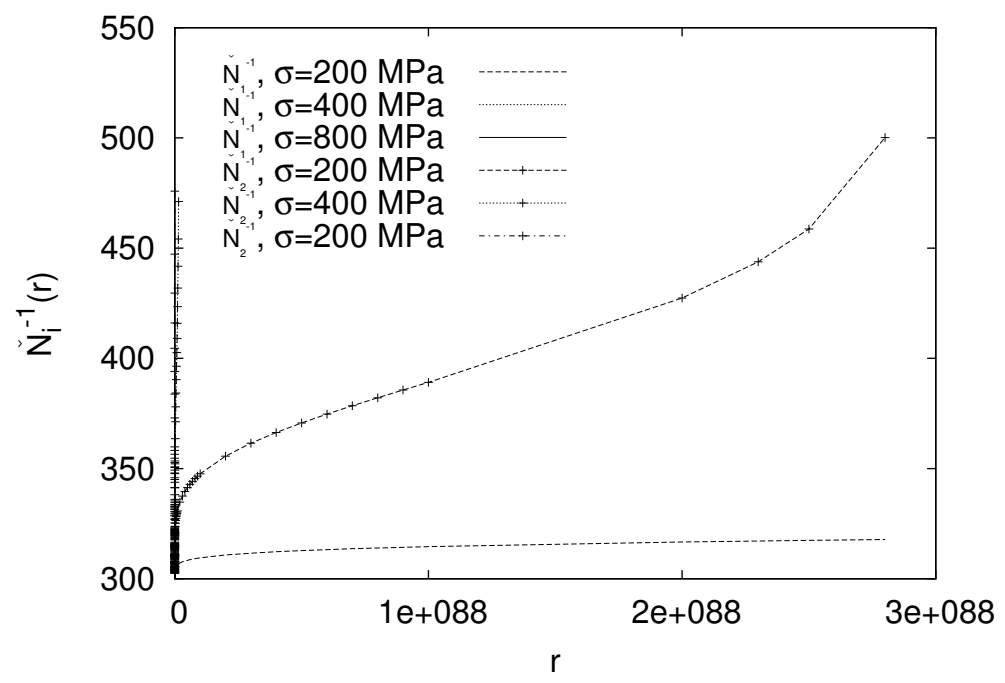

Figure 3: The functions $\left(\check{N}_{1}\right)^{-1}$ and $\left(\check{N}_{2}\right)^{-1}$ of the phases for different values of the steady stress $\sigma^{\star \star}$ (the curves seem to coincide for the three values of $\left.\sigma^{\star \star}\right)$

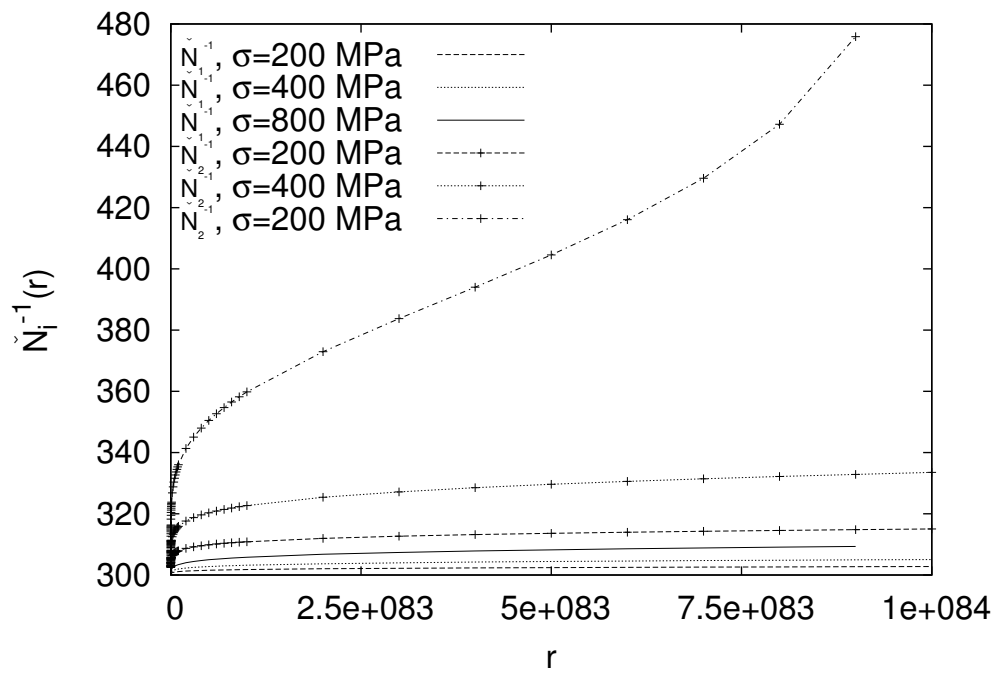

Figure 4: The function $\left(\check{N}_{1}\right)^{-1}$ and $\left(\check{N}_{2}\right)^{-1}$ of the phases for different values of the steady stress $\sigma^{\star \star}$ at the onset of the deformation (the curves differ for the three values of $\sigma^{\star \star}$ ) 


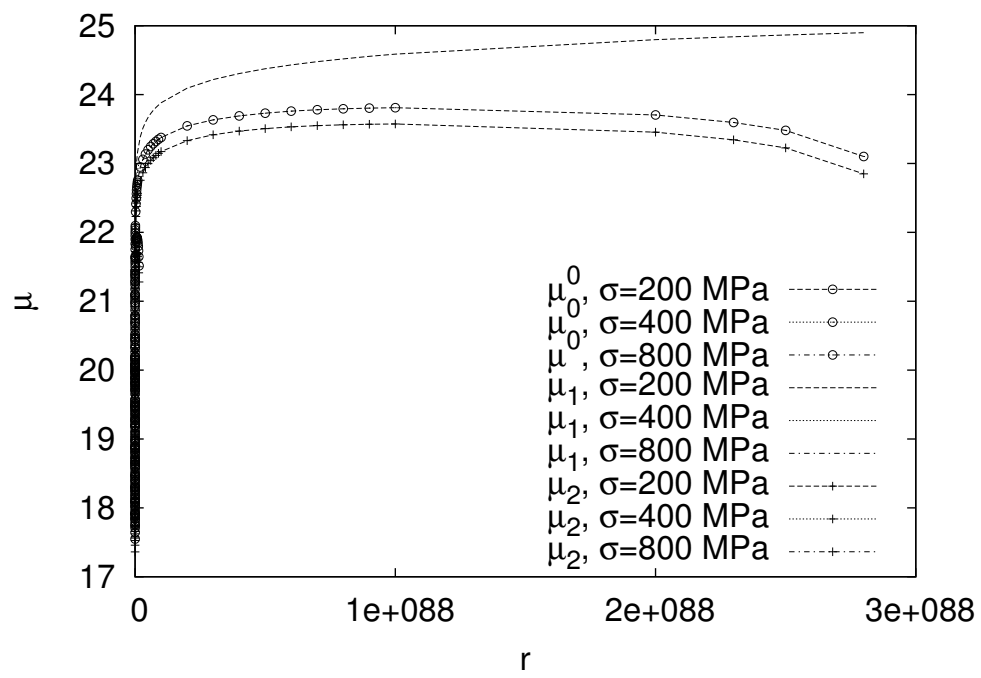

Figure 5: The softenings $\mu_{1}$ and $\mu_{2}$ of the phases and the homogenized softening $\mu^{0}$ for different values of the steady stress $\sigma^{\star \star}$ (the curves seem to coincide for the three values of $\sigma^{\star \star}$ )

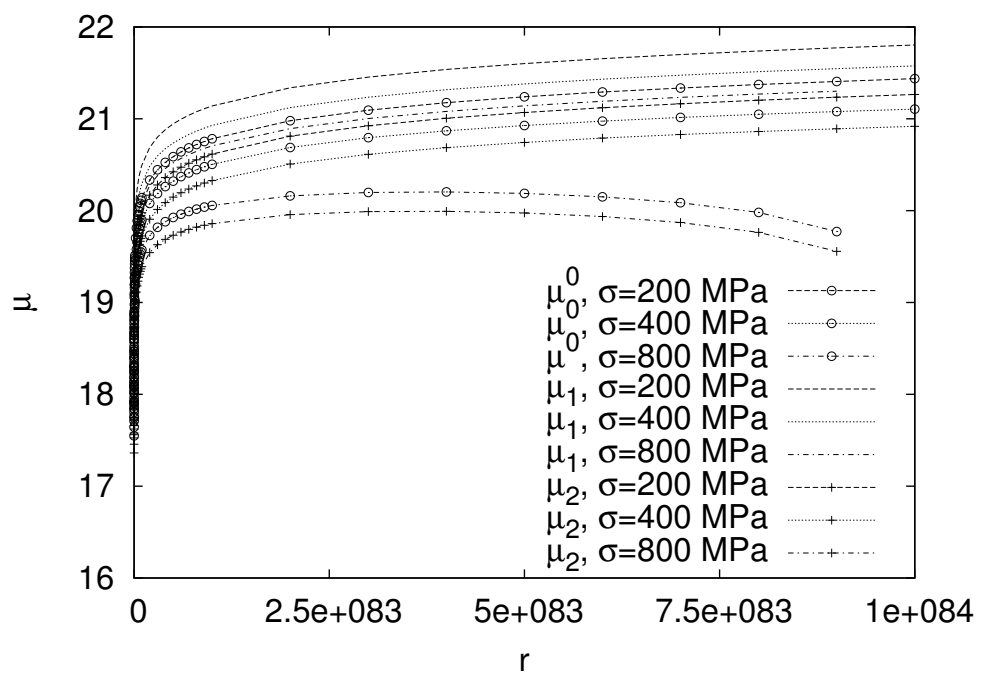

Figure 6: The softenings $\mu_{1}$ and $\mu_{2}$ of the phases and the homogenized softening $\mu^{0}$ for different values of the steady stress $\sigma^{\star \star}$ at the onset of the deformation (the curves differ for the three values of $\sigma^{\star \star}$ ) 


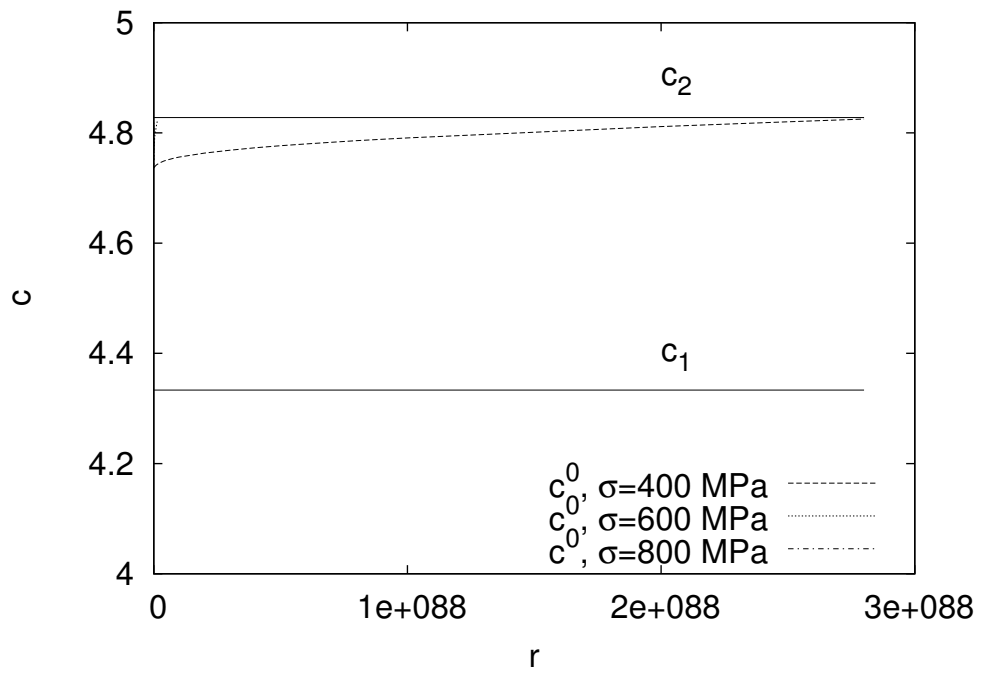

Figure 7: The (constant) heat coefficients $c_{1}$ and $c_{2}$ of the phases and the homogenized heat coefficient $c^{0}$ for different values of the steady stress $\sigma^{\star \star}$ (the curves seem to coincide for the three values of $\sigma^{\star \star}$ )

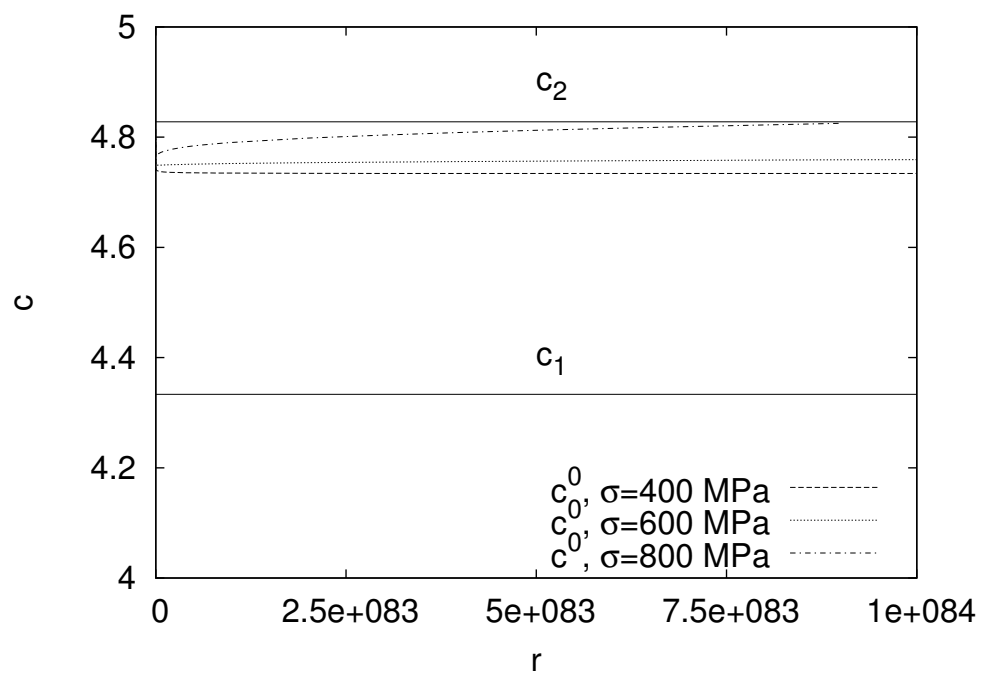

Figure 8: The (constant) heat coefficients $c_{1}$ and $c_{2}$ of the phases and the homogenized heat coefficient $c^{0}$ for different values of the steady stress $\sigma^{\star \star}$ at the onset of the deformation (the curves differ for the three values of $\sigma^{\star \star}$ ) 